\title{
Ecoepidemiology: a More Comprehensive View of Population Interactions
}

\author{
E. Venturino * \\ Dipartimento di Matematica "Giuseppe Peano" \\ Università di Torino, Italy
}

\begin{abstract}
Models proposed for the description of population interactions with pathogenic agents are presented. We review some basic quadratic-interactions models, then move to more complex demographics, structured models, models involving several populations, delays, plankton dynamics, the Allee effect and group behavior. In most of these cases basic reproduction numbers are evaluated and commented.
\end{abstract}

Keywords and phrases: ecoepidemics, food webs, Allee effect, plankton, delays, harvesting, age-dependent, stage-dependent, switching, metaecoepidemic, group behavior

Mathematics Subject Classification: 92D25, 92D40,

\section{Introduction}

Diseases are a fact in nature. They are caused by helmints, bacteria, viruses or fungi. Thus, when considering infected populations, a "hidden" infectious agent population is present in the model, whether explicitly or implicitly. On the other hand, populations interact in various ways, we can just think, for instance, of food webs, competition systems and cooperating organisms. Therefore, although in epidemiology one is interested in the disease outcome and possibly in its control, in reality mathematical models for epidemics are ultimately population models. It makes sense to consider population models in which some of the state variables are in fact pathogenous agents. When these appear in general models accounting for several other "biological", i.e. disease-free, populations, we deal with ecoepidemiological models, a natural extension of more classical population interactions systems, as remarked in Section 3.6.

In the mid eighties purely epidemic models started to depart from the classical assumption of constant population size, $[25,52,98]$. At the same time research on these topics began, $[4,17,60,129]$. Upon a suggestion of the late $\mathrm{O}$. Arino, the name to the field, ecoepidemics, was given by J. Chattopadhyay $[32,97,106]$.

The field since then has grown enormously, hence we give a caveat to the reader. In this presentation we confine essentially to deterministic models. Also, we do not consider the many models that include a spatial description of the environment, nor is the bibliography meant to be exhaustive, as many more papers than those indicated here appeared.

\footnotetext{
*Corresponding author. E-mail: ezio.venturino@unito.it
} 
The paper is organized as follows: we start with a brief review of the classical demographic and epidemic models, essentially to set the notation, followed by Section 3 on fundamental ecoepidemic models, presented through three basic examples, reformulations and an interpretation in terms of community models. These topics are dealt with in some detail, to explain the basic features and pave the road for the remaining topics. Section 4 contains models for which the demographics is more realistic. It may account for the observation that the daily uptake of food by predators is limited, or that a saturation phenomenon occurs when the prey is too much abundant, for which the predators would ignore it, if not very hungry. We also include models for an age description of the situation, be it the age of the individuals or the one of the pathogens, thereby specifying the stage of the disease. In Section 5 we move then to situations in which more populations are involved, looking at feeding switching, two disease strains, food chains, metaecoepidemic situations, chaotic behavior. Some models including delays are then illustrated in Section 6, followed by Section 7 on models for plankton dynamics. Finally, more recent investigations are contained in Section 8, including models for group behavior, the Allee effect, the concept of emerging carrying capacity, ecogenetic models.

\subsection{Notation}

Unless otherwise specified, the notation is as follows: $N$ denote the prey, divided into susceptibles $S$, infected $I$ and $R$ the recovered; for the predators $Z, P$ are the susceptibles, $Y$ the infected and $U$ the recovered. The respective prevalences, i.e. the proportion of disease-affected individuals with respect to the total population, are $i=I N^{-1}$ and $y=Y Z^{-1}$. The equilibria are denoted by $E_{k} \equiv\left(N_{k}, Z_{k}\right)$, where $M_{k}$ is the population size, $M \in\{N, Z\}$, and similarly for more populations.

For the parameters appearing in the model formulation, assumed to be nonnegative unless otherwise specified, generally greek letters have an epidemic significance, while latin letters denote demographic quantities. In view of the different notations used in the many models presented, we decided, however, to keep the original parameters and avoid to use a uniform notation, as that is prone to errors. Thus there might be some occasional departures from the above convention. We expect that the reader, after the initial examples, can easily understand their meaninings, if not explicitly specified.

\section{Background}

Let us start by considering the two classical models in interacting populations and in epidemiology.

\subsection{Demographics}

The classical quadratic predator-prey model, see e.g. [97,105,142], is given by

$$
N^{\prime}=N[a-b N-c Z], \quad Z^{\prime}=Z[d+e N-f Z] .
$$

The parameters are assumed to be nonnegative, unless otherwise stated. The prey grow logistically with carrying capacity $K=a b^{-1}$ and intrinsic birth rate $a$ :

$$
N^{\prime}=N[a-b N]=a N\left(1-N K^{-1}\right) .
$$

Further, $c$ is the killing rate due to predation, which occurs giving a return rate $e<c$ to the predators, as not the whole prey captured is converted into predators' newborns biomass. Note that in the absence of the prey, the predators still thrive, and tend to the value $d f^{-1}, d$ being their reproduction rate due to resources other than the prey $N, f$ their intraspecific competition rate. Thus, here the predators are generalist, they do not feed just on this specific prey population as it occurs in the classical Volterra model, where $d=-m$ is instead a mortality. In fact, the latter model was conceived to describe the shark-fish interactions in the Adriatic sea. In this environment, the largest predators must be specialists as they have no food source other than the fish. 
Coexistence is an always locally asymptotically stable equilibrium, $P_{4} \equiv\left[(a f-c d) D^{-1},(a e+b d) D^{-1}\right]$ with $D=b f+c e$. It is feasible when the "invasion coefficient" for the predators exceeds one:

$$
R_{p} \equiv \frac{a f}{c d} \geq 1
$$

and globally asymptotically stable. The other equilibria are the system disappearance $P_{1} \equiv(0,0)$ and the single population point $P_{3} \equiv\left(a b^{-1}, 0\right)$, which are saddles, the prey-only point $P_{2} \equiv\left(0, d f^{-1}\right)$ which is stable if and only if (2.3) fails, namely for $R_{p}<1$. i.e. when the nontrivial equilibrium becomes unfeasible, showing a transcritical bifurcation. Other classical types of demographic models are symbiotic and competing systems, see e.g. [142]. In all these quadratic systems, persistent oscillations are shown to be impossible.

Another classical predator-prey model leading instead to limit cycles is the Rosenzweig-MacArthur model, [108]:

$$
N^{\prime}=b N\left(1-\frac{N}{K}\right)-\frac{a N}{H+N} Z, \quad Z^{\prime}=e \frac{a N}{H+N} Z-d Z
$$

where $b$ is the net prey birth rate, $K$ their carrying capacity, $a$ their removal rate by predators hunting, $e<1$ is the conversion coefficient of captured prey into predators newborns, $H$ the half saturation constant and $d$ the predators mortality.

\subsection{Epidemics}

Assuming a large constant population, $M$, partitioned among susceptibles and the infectives $S$ and $I$ respectively, the classical SIS epidemic model is, [73,97]:

$$
S^{\prime}=-\lambda(M) I S+\gamma I+\mu M-\mu S, \quad I^{\prime}=\lambda(M) I S-\gamma I-\mu I .
$$

Here $\gamma$ denotes the disease recovery rate. The parameter $\mu$ instead stands for the mortality rate which is equal to the birth rate; this is expressed by the third term in the right hand side of the first equation, which also says that the disease is not vertically transmitted, as the newborns of the whole population, susceptible plus infected, are born healthy. The horizontal disease transmission is modeled by the $\lambda$ function while the recovery rate is $\gamma$. The incidence is usually assumed to be in either one of the following forms representing respectively mass action incidence and standard incidence:

$$
\lambda(M)=\lambda ; \quad \lambda(M)=\lambda M^{-1} .
$$

The first one is usually adopted with the model (2.5), while the second one is employed with an alternative formulation of (2.5) that replaces $S$ and $I$ by the fractions of the total population size, $s=S M^{-1}, i=I M^{-1}$, in which case $s+i=1$.

A more general SIRS model could be formulated, if infected are recognized as disease-carriers, so that they are isolated to prevent further contagions. For a brief introduction of these topics see [97], or, for a comprehensive review, also [73].

\section{Ecoepidemiological models: the basics}

To obtain an ecoepidemic model, $[129,130]$, we combine the above systems introducing for instance a transmissible nonfatal disease in the prey. We assume that only susceptible prey are capable of reproduction, while infected are too weak even to exert any intraspecific competition. Predators hunt both types of prey at different rates. 


\subsection{The first example: disease among the prey}

The model is constructed merging (2.1) and (2.5), and assuming the first form of (2.6), [130], thus obtaining:

$$
S^{\prime}=S[a-b S-c Z-\lambda I]+\gamma I, \quad I^{\prime}=I[\lambda S-k Z-\gamma], \quad Z^{\prime}=Z[d+e S-f Z-h I] .
$$

For $h>0$ interactions lead to death of predators upon contact with an infected prey; if $h<0$, the predators do not get harm and hunting on infected animals occurs at a different, larger $h \geq e$, rate than on sound prey: for instance, infected fish come closer to the sea surface becoming an easier prey for birds.

The origin $E_{0} \equiv(0,0,0)$ and the healthy prey-only point $E_{1} \equiv\left(a b^{-1}, 0,0\right)$ are both unstable. The predators-only equilibrium, existing due to the other food sources, is $E_{2} \equiv\left(0,0, d f^{-1}\right)$. It is locally asymptotically stable whenever (2.3) does not hold, $R_{p}<1$, i.e. the predators invasion coefficient is below the critical threshold as for the purely demographic model. These demographic equilibria are thus unaffected by the presence of the disease.

Coexistence in a disease-free environment, $E_{3} \equiv\left[(a f-c d) D^{-1}, 0,(d b+e a) D^{-1}\right]$, is feasible when (2.3) holds. Its projection onto the "demographic" $S-Z$ plane coincides with the equilibrium $P_{4}$ of the underlying demographic model, explaining the reason for the very same feasibility condition. For the stability analysis however, one eigenvalue is $\lambda S_{3}-\left(k Z_{3}+\gamma\right)$; the other ones have negative real parts, since the Routh-Hurwitz conditions of the remaining $2 \times 2$ minor $J_{2 \times 2}$ of the Jacobian are satisfied, $-\operatorname{tr}\left(J_{2 \times 2}\right)=b S_{3}+f Z_{3}>0, \operatorname{det}\left(J_{2 \times 2}\right)=D S_{3} Z_{3}>0$. Thus stability hinges just on the first eigenvalue:

$$
(a f-d c) \lambda<k(d b+e a)+\gamma D .
$$

From the presence of the disease contact and recovery rates in this condition it is clearly seen that the disease may severely affect the demographic behavior of the system, preventing coexistence from occurring. Note that no endemic predator-free equilibrium of the form $(+,+, 0)$ exists because in this case $I \rightarrow \infty$.

Coexistence is attained at the point $E_{4}$ with populations $Z_{4}=\lambda S_{4} k^{-1}, I_{4}=-\theta \Delta^{-1}, S_{4}=k \Psi \Delta^{-1}$, where

$$
\Delta \equiv \lambda(\lambda f-h c)-k(b h+\lambda e), \quad \Psi \equiv \lambda d-a h, \quad \theta \equiv \lambda(d c-f a)+k(d b+e a) .
$$

The feasibility conditions are either the set:

$$
\Psi>0, \quad \Delta>0, \quad \theta<0,
$$

or the set of opposite inequalities. The characteristic polynomial is $\Xi(\mu)=\mu^{3}+a_{1} \mu^{2}+a_{2} \mu+a_{3}$, with

$$
a_{1}=\Psi(f \lambda+k b) \Delta^{-1}, \quad a_{2}=k \lambda \Psi[\theta(h-\lambda)+\Psi D] \Delta^{-2}, \quad a_{3}=-k \lambda \theta \Psi^{2} / \Delta^{-2} .
$$

For the Routh Hurwitz conditions we find that by (3.3) both $a_{1}>0$ and $a_{3}>0$ hold. The stability requirement reduces just to $a_{1} a_{2}>a_{3}$. Explicitly:

$$
(f \lambda+b k)[D \Psi+\theta(h-\lambda)]+\theta \Delta>0 .
$$

By observing that the last two terms become

$$
\theta[(f \lambda+b k)(h-\lambda)+\Delta] \equiv \theta \lambda[h(f-c)-k(b+e)],
$$

for the case $h<0$ requiring $f>c$ then implies (3.4). The opposite set of inequalities of (3.3) implies $a_{3}>0$ which contradicts $\theta>0$ and therefore leads to an unstable equilibrium.

For models based on other demographic interactions see [131,134]. 


\subsection{Reformulation in terms of disease prevalence}

In fairly recent papers, $[78,80,103,106,119]$, similar models are analysed via a dependent variable reformulation in terms of total prey population and disease prevalence, recall the definition $i=I N^{-1}$ given in the Notation Section 1.1, allowing to distinguish disease-induced extinction from the trivial equilibrium. If this perspective is taken on (3.1), elementary calculations give

$$
N^{\prime}=a S-b S^{2}-c Z S-k Z I, \quad i^{\prime}=i\left[\lambda S-k Z-\gamma-S N^{-1}(a-b S-c Z)+k Z i\right]
$$

and back substitution into (3.1) provides the system's reformulation in the $i N Z$ phase space

$$
\begin{aligned}
i^{\prime} & =i[\lambda N(1-i)-k Z-\gamma-(1-i)[a-b N(1-i)-c Z]+k i Z], \\
N^{\prime} & =N\{(1-i)[a-b N(1-i)-c Z]-k i Z\}, \quad Z^{\prime}=Z[d+e N(1-i)-f Z-h i N] .
\end{aligned}
$$

The disease-free equilibria are now $E_{0}^{*}=(0,0,0), E_{1}^{*}=\left(0, a b^{-1}, 0\right), E_{2}^{*}=\left(0,0, d f^{-1}\right)$ and $E_{3}^{*}=$ $\left(0,(a f-c d) D^{-1},(a e+b d) D^{-1}\right)$. The first two are again unstable, but the predator-only equilibrium $E_{2}^{*}$ is stable under different conditions than for $E_{2}$, namely we need to require, in addition to $R_{p}<1$, also $R_{p}>1-(k d+\gamma f)(c d)^{-1}$. The point is that the two equilibria $E_{1}$ and $E_{1}^{*}$ do not provide the same information, as the first one is the predator-only equilibrium, while $E_{1}^{*}$ says something more than what is expressed by $E_{1}$. In fact, it represents the predator-only equilibrium in which the whole prey population disappears, but in doing so, the disease has already been eradicated, as its prevalence vanishes. The prey could instead disappear bearing endemically the disease within its population, so that the infected must also go to zero, and that could happen at equilibrium $E_{1}^{*}$.

Following [106], we can write also the Jacobian of (3.5) at the disease-free equilibrium $E_{3}^{*}$ :

$$
\left(\begin{array}{ccc}
\lambda N_{3}^{*}-k Z_{3}^{*}-\gamma & 0 & 0 \\
N_{3}^{*}\left(b N_{3}^{*}\left(1-2 i_{3}\right)-k Z_{3}\right) & -b N_{3}^{*}-c N_{3}^{*} \\
-(e+h) N_{3}^{*} Z_{3}^{*} & e Z_{3}^{*} & -f Z_{3}^{*}
\end{array}\right)
$$

so that the $2 \times 2$ bottom right minor corresponds to the Jacobian of the purely demographic model (2.1), for which the Routh-Hurwitz conditions are satisfied. The Jacobian is partitioned, and the top left entry determines the stability of this disease-free equilibrium, giving once again the stability condition (3.2) as for the original model (3.1). The effects of the diseased prey respectively on the healthy prey and predator populations are contained in the bottom portion of the first column.

\subsection{The second example: disease among the predators}

As a comparison, we can discuss briefly also the predator-prey model with the disease affecting the predators. The model with infection in the predators can be formulated as follows, [132]:

$$
\begin{aligned}
& N^{\prime}=N[a-b N-c P-\eta Y], \quad P^{\prime}=P[d+e N-f(P+Y)-\delta Y]+\nu Y, \\
& Y^{\prime}=Y[\delta P+g N-f(P+Y)-\nu] .
\end{aligned}
$$

Introducing the total predators population and their disease prevalence, recall once more the definition $y=Y Z^{-1}, Z=P+I$, given in the Notation Section 1.1, using the shorthand notation $w=1-y$, the above system becomes:

$$
N^{\prime}=N[a-b N-c Z w-\eta y Z], \quad Z^{\prime}=Z[g y N+(d+e N) w-f Z], \quad y^{\prime}=y[w(\delta Z+(g-e) N-d)-\nu] .
$$

The origin $P_{1}$, the predator-and-disease-free equilibrium $P_{3}=\left(a b^{-1}, 0,0\right)$ and the prey-only point with the disease endemically affecting the whole predator population $P_{5}=\left(a b^{-1}, 0,1-\nu\left[(g-e) a b^{-1}-d\right]^{-1}\right)$ 
are always unstable equilibria. The healthy predators-only equilibrium $P_{2}=\left(0, d f^{-1}, 0\right)$ can be stably achieved, in agreement with the results in [132] for (3.6), whenever

$$
\frac{a}{c}<\frac{d}{f}<\frac{d+\nu}{\delta}
$$

The prey-free equilibrium $P_{4}=\left(0, d f^{-1} h, 1-h\right)$ with $h=f(d+\sqrt{\Delta})(2 \delta d)^{-1}, \Delta=d^{2}+4 \nu \delta d f^{-1}$, is feasible when the second condition $(3.8)$ is reversed and stable if

$$
a<c Z_{4}\left(1-y_{4}\right)+\eta Z_{4} y_{4}
$$

Finally coexistence in a disease-free environment, i.e. the purely demographic coexistence, given by $P_{6}=\left((a f-c d) D^{-1},(a e+b d) D^{-1}, 0\right)$, with $D \equiv c e+b f$ is feasible for $R_{p} \geq 1$, compare (2.3). Note that in this case, as for the model (3.1), at this point the Jacobian is a partitioned matrix:

$$
\left[\begin{array}{ccc}
-b N_{6}-c N_{6} & (c-\eta) Z_{6} N_{6} \\
e Z_{6} & -f Z_{6} & (g-e) Z_{6} N_{6}-d Z_{6} \\
0 & 0 & \delta Z_{6}+(g-e) N_{6}-d-\nu
\end{array}\right]
$$

where the bottom right entry gives the stability condition of this equilibrium:

$$
\delta Z_{6}+(g-e) N_{6}<d+\nu .
$$

Also, the coexistence equilibrium with endemic disease is attainable via numerical simulations. By comparing the second condition (3.8) with feasibility for $P_{4}$ and the first condition (3.8) with (2.3), transcritical bifurcations are seen to occur between the pair of points $P_{2}$ and $P_{4}$, as well as among $P_{2}$ and $P_{6}$.

\subsection{The third example: a more complex model}

An SIRS model is instead presented in [7]. Introducing the class of recovered individuals in the predators $Z=P+Y+U$, produces the system

$$
\begin{array}{ll}
N^{\prime}=\varepsilon\left[r N\left(1-N K^{-1}\right)-a N(P+Y+U)\right], & P^{\prime}=(\gamma U-\beta P Y)+\varepsilon[-\mu P+b N P], \\
Y^{\prime}=(\beta P Y-\delta Y)+\varepsilon\left[-\mu Y-\mu^{*} Y+b N Y\right], & U^{\prime}=(\delta Y-\gamma U)+\varepsilon[-\mu U+b N U],
\end{array}
$$

where $N$ is the prey population, $\mu^{*}$ denotes the disease-related mortality and $\delta$ is the progress rate of individuals from infected to recovered. Assuming that the disease has a faster dynamics than the demographic processes, the aggregation method can be used to render (3.11) more manageable. Assuming that the fast dynamics quickly settles to equilibrium, the aggregated system attains either one of the following forms, respectively in the case $\beta p<\delta$ and conversely:

$$
\begin{array}{ll}
N^{\prime}=N\left[r\left(1-N K^{-1}\right)-a Z\right], & Z^{\prime}=Z(b N-\mu) \\
N^{\prime}=N\left[r\left(1-N K^{-1}\right)-a Z\right], & Z^{\prime}=b N Z-\mu \gamma(\gamma+\delta)^{-1}\left(Z-\delta \beta^{-1}\right)-\mu Z .
\end{array}
$$

Coexistence equilibria in both slow dynamics are found to be globally asymptotically stable, respectively $\left(\mu b^{-1}, r a^{-1}\left(1-\mu(b K)^{-1}\right)\right)$ and $\left(N_{2}^{*}, Z_{2}^{*}\right)$, with $N_{2}^{*}=K\left(1-a r^{-1} Z_{2}^{*}\right)$ and

$$
Z_{2}^{*}=\frac{r}{a} \frac{\sqrt{\Delta}-\beta\left[(\gamma+\delta)(\mu-b K)+\mu^{*} \gamma\right]}{2 b \beta K(\gamma+\delta)}, \quad \Delta=\beta^{2}\left[(\gamma+\delta)(\mu-b K)+\mu^{*} \gamma\right]^{2}+\frac{4}{r} a b K \beta \mu^{*} \delta \gamma(\gamma+\delta) .
$$




\subsection{Use of the standard disease incidence}

In the form of total prey population, prey prevalence and predators formulation, the model of [75] containing disease contagion modeled via standard incidence is:

$$
\begin{aligned}
& N^{\prime}=N\left[r\left(1-\frac{N}{K}\right)-a[1-(q-1) i] Z\right], \\
& i^{\prime}=i\left[\beta(1-i)-\left(\gamma+b-\frac{\theta r N}{K}\right)-a(q-1)(1-i) Z\right], \quad Z^{\prime}=Z[k a N[1+(q-1) i]-c] .
\end{aligned}
$$

Six possible behaviors are identified depending on the following quantities:

$R_{0}=\frac{\beta}{\gamma+b-\theta r}, \quad R_{1}=\frac{\beta}{\left[\gamma+b-\theta r(k a K)^{-1}\right](q-1) r\left[1-c(k a K)^{-1}\right]}, \quad \psi=(q-1) \frac{R_{0}-1}{R_{0}}-\left[\frac{c}{k a K}-1\right]$.

Assume at first that $k a K \leq c$ : for $R_{0} \leq 1$ the system attains the healthy-prey-only equilibrium, for $R_{0}>1$ and $\psi<0$ the predators disappear and the disease is endemic among the prey. When $R_{0}>1$ and $\psi>0$ instead the system behavior toward coexistence is conjectured.

Take now $k a K>c$ : in both cases $R_{1}<R_{0} \leq 1$ and $R_{1} \leq 1<R_{0}$ the disease gets eradicated, in the latter case this occurs due to the predation of the more vulnerable infected prey, in the absence of predators the disease would remain endemic; finally for $1<R_{1}<R_{0}$ the system should attain coexistence with endemic disease. Conditions are also identified, both for endemicity of the disease, $k a K>c$ and $R_{1}>1$, as well as for system persistence, $R_{0}<1$ and $k a K>c$, or, alternatively, $R_{0}>1$ together with $\psi>0$.

Standard incidence and mass action transmission are also compared in the model of [103], which in nondimensional form is:

$$
N^{\prime}=N(1-N)-N Z, \quad i^{\prime}=i[(\Gamma(Z)-\alpha)(1-i)-e N], \quad Z^{\prime}=Z[e N-m-\alpha i] .
$$

Here $\Gamma(Z)$ is the disease transmission term, represented by either mass action or standard incidence. Relevant reproduction numbers are introduced, namely the predators' basic reproduction number in the absence of the disease, the disease basic reproduction number, the predators' basic reproduction number in the presence of the disease and the prevalence reproduction number, defined only for the standard incidence model, respectively:

$$
R_{1,0}^{Z}=e m^{-1}, \quad \mathcal{R}_{0}=\Gamma\left(Z^{*}\right)(m+\alpha)^{-1}, \quad R_{1, i}^{Z}=e\left(m+\alpha i^{*}\right)^{-1}, \quad \mathcal{R}_{i}=\mathcal{R}_{0}\left(R_{1, i}^{Z}\right)^{-1} .
$$

Here $Z^{*}$ and $i^{*}$ represent respectively the predators population size at the disease-free equilibrium and the prevalence when the disease becomes endemic.

The mass action model settles to the prey-only equilibrium $(1,0,0)$ for $R_{1,0}^{Z}<1$, to the disease-free equilibrium when both conditions $R_{1,0}^{Z}>1$ and $\mathcal{R}_{0}<1$ are satisfied, to the coexistence with endemic disease point or persistent oscillations around it whenever $R_{1,0}^{Z}>1$ and $\mathcal{R}_{0}>1$ both hold.

The standard incidence system instead exhibits one more equilibrium, the predator-free equilibrium with endemic disease, whenever $R_{1,0}^{Z}>1, \mathcal{R}_{0}>1$ and $R_{1, i}^{Z}<1$, or, alternatively, both $R_{1,0}^{Z}<1$ and $\mathcal{R}_{i}>1$ hold. In this model the prey-only equilibrium is attained for $R_{1,0}^{Z}<1$ and $\mathcal{R}_{i}<1$, the disease-free point whenever $R_{1,0}^{Z}>1$ and $\mathcal{R}_{0}<1$ hold, the endemic coexistence equilibrium, or limit cycles around it, for $R_{1,0}^{Z}>1, \mathcal{R}_{0}>1$ and $R_{1, i}^{Z}>1$.

\subsection{Interpretation as community models}

In [119] the similarity in structure and behavior of food chain, exploitative competition (EC) and intraguild predation (IGP) models is explored. In the latter, the IG predator hunts an IG prey, and both have a common prey at the bottom level. Thus, also ecoepidemic models can be accounted for in this 
class. In EC models instead, not all the three species directly interact with each other. The relationship between these structures is evidenced by a suitable coordinate transformation. The ecoepidemic model, which corresponds to IGP, is, [119]:

$$
\begin{aligned}
& S^{\prime}=r S[1-(S+I)] S-a \frac{S Z}{h+S+I}-\lambda \frac{S I}{S+I}, \\
& I^{\prime}=I\left(\lambda \frac{S}{S+I}-a \frac{Z}{h+S+I}-\mu\right), \quad Z^{\prime}=Z\left(\varepsilon a \frac{S+I}{h+S+I}-m_{Z}\right) .
\end{aligned}
$$

With the coordinate transformation $N=S+I, v=I S^{-1}$, note that this is the ratio of infected to susceptibles, not the disease prevalence, the same transformation used later in [106], we obtain the transformed model:

$$
\begin{aligned}
& N^{\prime}=N\left[\frac{r}{1+v}(1-N)-\mu \frac{v}{1+v}-a \frac{P}{h+N}\right], \\
& v^{\prime}=v[r N-(\mu+r-\lambda)], \quad Z^{\prime}=Z\left(\varepsilon a \frac{N}{h+N}-m_{Z}\right) .
\end{aligned}
$$

This model resembles very closely the EC community module presented in [6], namely:

$$
A^{\prime}=A\left[r(1-A)-a_{1} B-a_{2} \frac{V}{h+A}\right], \quad B^{\prime}=B\left(\varepsilon_{1} a_{1} A-m_{1}\right), \quad V^{\prime}=V\left(\varepsilon_{2} a_{2} \frac{A}{h+A}-m_{2}\right),
$$

where $A$ is a prey and $B$ and $V$ two consumers. If the disease is in the predator (rather than in the prey) population, the model can be transformed, under certain conditions, into a food chain (rather than into an exploitative competition) community module [119]. This food chain has $V$ as the top predator, $B$ at the intermediate level and $A$ is the bottom prey.

\section{More realistic demographics}

Demographic models better fitting reality can be constructed by observing that the daily uptake of food by predators is limited, or that a saturation phenomenon occurs when the prey is too much abundant, for which the predators would ignore it, if not very hungry; alternatively, there could be a kind of carrying capacity for predators that depends on the size of the prey population. Further, we could better describe individuals, even pathogens, by their biological age.

As we will see, the Leslie-Gower and the Holling-Tanner models exhibit very similar qualitative properties. The latter has one more parameter available and therefore exhibits somewhat more complicated conditions characterizing the equilibria. Moreover, the additional nonlinearity in it induces one extra possible equilibrium.

\subsection{The model with feeding satiation}

This situation is generally modeled by introducing the Holling type II response function, with a horizontal asymptote. This idea has been exploited also in the context of ecoepidemiology, [32], replacing (3.1) by

$$
\begin{aligned}
& S^{\prime}=r(S+I)\left[1-\frac{S+I}{k}\right]-b S I-\frac{\eta m_{1} S Z}{a_{1}+S}, \quad I^{\prime}=I\left[b S-\frac{m Z}{a+I}-c\right], \\
& Z^{\prime}=\left[\frac{\varepsilon m I}{a+I}+\frac{\eta \varepsilon m_{1} S}{a_{1}+S}-d\right] Z .
\end{aligned}
$$

In (4.1) also the infected can reproduce and give birth to healthy individuals, the disease thus is not transmitted vertically. The introduction of the nonlinearities of Holling type II leads to rather different results than those obtained in model (3.1). In addition to global stability results for some equilibria, 
as well as transcritical bifurcations, the most important finding is that there is a supercritical Hopf bifurcation, for the predation's hunting rate on healthy prey lying in a suitable window:

$$
d+a b d(b k-c)^{-1}<\varepsilon m<\min \left\{d(1-b)^{-1}, a b(a b d)^{\frac{1}{4}}+d-3 a d\right\} .
$$

The model (4.1) has value in that it represents another one of the earliest contributions in this field. With the current knowledge, however, this formulation presents some drawbacks. In fact, the predator feeds on both healthy and infected prey, but according to the equations, it may experience satiation of the former independently of the latter, or vice versa. These assumptions are debatable because the predator would then be able to distinguish among the two types of prey, and make a choice in hunting. This represents a possible but perhaps unlikely alternative. A viable alternative would instead be to use a Beddington-De Angelis response function, $[16,46]$, that incorporates satiation for both types of prey in a single term. This view is in fact taken in several of the most recent contributions, see the models that we present below, for instance (7.7) of [123] or (7.9) of [78].

Note that if the disease were to affect only the predators, this problem would not sussist. In fact, another system with feeding satiation in which the underlying demographics is represented by the Rosenzweig-McArthur dynamics (2.4) is presented in [80]. In it also the mass action incidence is replaced by the standard incidence, corresponding to proportional mixing, or also to frequency dependence. Here, the disease affects the predators, the prey $X(T)$ are not affected:

$$
X^{\prime}=b X \frac{K-X}{K}-\frac{a X(P+Y)}{H+X}, \quad P^{\prime}=\frac{e a X(P+Y)}{H+X}-d P-\frac{\sigma P Y}{P+Y}, \quad Y^{\prime}=\frac{\sigma P Y}{P+Y}-(d+\alpha) Y ;
$$

note the disease-related mortality $\alpha$. Disease prevalence $i=Y(P+Y)^{-1}=y Z^{-1}$ is introduced, removing the system singularity when the predators population vanishes. Rescaling via the substitutions $N(t)=$ $X K^{-1}, Z(t)=(P+Y)(e K)^{-1}, t=e a T$ and letting $r=b(e a)^{-1}, m=d(e a)^{-1}, h=H K^{-1}, \beta=\sigma(e a)^{-1}$, $\mu=\alpha(e a)^{-1}$ leads to the nondimensional model with two healthy populations and disease prevalence:

$$
N^{\prime}(t)=r N(1-N)-\frac{N Z}{h+N}, \quad Z^{\prime}(t)=\frac{N Z}{h+N}-m Z-\mu Z i, \quad i^{\prime}(t)=\left[(\beta-\mu)(1-i)-\frac{N}{h+N}\right] i .
$$

The advantages of the reformulation appear in the possibility of identifying four thresholds that allow to describe the ecosystem composition. More precisely, these reproduction numbers are:

$$
R_{0}^{p}=\frac{1}{m(1+h)}, \quad \mathcal{R}_{0}=\frac{\beta}{m+\mu}, \quad R_{i}^{p}=\frac{\beta}{(\beta-\mu)(m+\mu)(1+h)}, \quad \mathcal{R}_{i}=\frac{\mathcal{R}_{0}}{R_{i}^{p}}=(\beta-\mu)(1+h) .
$$

In [80] they are called respectively the disease-free demographic, the epizootiological, the enzootic demographic and the prevalence reproduction numbers. The disease-free demographic reproduction numbers gives the expected number of offsprings of a predator in its lifetime, while the enzootic demographic reproduction number of predators $R_{i}^{p}$ accounts for the extra disease-related mortality in the presence of infection. The epizootiological reproduction number gives the usual average number of secondary infections produced by a single infectious individual introduced into a totally susceptible population. The prevalence reproduction numbers provides the number of secondary infections discounted by the fact that the population size changes in time. The system's behavior is characterized as follows: the prey-only state is achieved for $R_{0}^{p}<1$ and $\mathcal{R}_{i}<1$; the prey-only state with vanishing predators but with endemic disease is stable instead for for $R_{0}^{p}<1$ and $\mathcal{R}_{i}>1$, or, alternatively for $R_{0}^{p}>1, R_{0}^{i}<1$ and $\mathcal{R}_{0}>1$. The disease-free predator-prey equilibrium, whether stable or with limit cycles around it, is achieved for $R_{0}^{p}>1$ and $\mathcal{R}_{0}<1$; the predator-prey point with endemic disease, exists, again stably or with persistent oscillations, whenever $R_{0}^{p}>1, R_{p}^{i}>1$ and $\mathcal{R}_{0}>1$. The analysis further shows that the persistent oscillations of the purely demographic model can be stabilized by a high enough disease transmissibility. 


\subsection{Ecoepidemics with Leslie-Gower dynamics}

In [71] the following model is considered with an underlying Leslie-Gower predator-prey dynamics, for which the predator's growth is limited through the availability of prey. The latter represents thus a variable carrying capacity. In nondimensional form it reads:

$$
S^{\prime}=S(1-S)-S Z-\lambda S I, \quad I^{\prime}=\lambda S I-\eta I Z-\gamma I, \quad Z^{\prime}=Z\left[\delta-\frac{\beta Z}{S+I}\right] .
$$

The main results are the global asymptotical stability both of the disease-free equilibrium when it is locally asymptotically stable, which occurs when the disease contact rate falls below the threshold $\lambda<$ $\lambda^{[1]} \equiv \gamma+\delta \beta^{-1}(\eta+\gamma)$ and of the endemic coexistence equilibrium when the above threshold is exceeded. But, in addition, the infected removal rate by predation has to be sufficiently high, namely $\eta>1$. Note that for $\lambda<\lambda^{[1]}$ a transcritical bifurcation between the two above equilibria occurs.

The disease-free equilibrium is the counterpart of the coexistence equilibrium in the purely demographic Leslie-Gower model, where however it is globally asymptotically stable. Here it can be destabilized by the presence of the disease. The predators and prey are intrinsically persistent in the ecosystem. This however does not happen in other models, for instance in the case of competing populations, we find that one of the populations can vanish while the other one survives with endemic disease, [131].

The prevalence at the endemic equilibrium $E_{+}=\left(S_{+}, I_{+}, Z_{+}\right)$is $p(\lambda, \beta, \eta, \gamma, \delta)=[\beta(\lambda-\gamma)-(\eta+$ $\gamma) \delta]\left[\beta(\lambda-\gamma+\lambda \gamma]^{-1}\right.$, so that it decreases if $\eta$ or $\delta$ increase, i.e. the predators' net birth rate or their hunting rate on infected prey, relative to the healthy ones, increase. By differentiation, prevalence gets reduced also with increasing contact rate $\lambda$ and with predators intraspecific pressure $\beta$, if $\lambda(1+\gamma)<\gamma$.

Comparing the ecosystem total population at the two equilibria, at disease-free equilibrium it has the nondimensionalized value 1 , while at endemic coexistence it is $D^{-1}[\beta(\beta+\delta)(\lambda \gamma+\lambda-\gamma)]$ with $D=\lambda^{2} \beta+\delta(\eta+\lambda-\eta \lambda)>0$ to ensure nonnegativity of $S_{+}$. The latter is larger then if and only if $(1-\lambda) I_{+} D \beta^{-1} \geq 0$. Thus if the disease contact rate falls below one, the environment's disease-free total population is larger than the same situation with endemic disease.

Persistence and equilibria global stability appear in [115] for this ecosystem extension with intraspecific competition due to infected predators and of a satiation effect modeled by HTII nonlinearities.

In [57] a Leslie-Gower model with the predators selectively feeding only on infected prey is presented:

$$
S^{\prime}=r S\left(1-\frac{S}{K}\right)-\lambda S I, \quad I^{\prime}=\lambda S I-\frac{c I Z}{m Z+I}-\gamma I, \quad Z^{\prime}=\delta Z\left(1-\frac{h Z}{I}\right) .
$$

A further difference with respect to (4.4) is here represented by the second term in the infected dynamics. It is a ratio-dependent response function, see Section 4.4, modeling the predators' serious efforts in hunting. It states that the per capita predator growth rate depends on the ratio of the prey to predator populations.

The results of the analysis show uniform persistence of the healthy prey population: it is bounded away from zero at any time. The possibly stable system's outcomes are the healthy-prey-only point and the coexistence equilibrium with endemic disease. Persistent oscillations are triggered for a high enough disease contact rate, $\lambda>\lambda_{H}$, the latter being the root of the quadratic $\delta^{*} \lambda^{2}=b_{1} \lambda+b_{0}$, with $\delta^{*}=c h(m+h)^{-2}, \lambda_{0}=K^{-1}\left[\gamma+c(m+h)^{-1}\right], b_{1}=\left(r+\delta^{*}\right) \lambda_{0}+\left(\delta-\delta^{*}\right)^{2} K^{-1}, b_{0}=r \lambda_{0}\left[\left(\delta-\delta^{*}\right) K^{-1}-\lambda_{0}\right]$.

A generalisation of (4.4) is presented in [114], in which the presence of the infected class is felt both by healthy as well as infected prey, in that it contributes to the intraspecific competition, with predators capturing both kinds of prey at different rates. Here the basic reproduction number $R_{0}$ depends inversely also on the intraspecific parameters, so that the latter exert a positive role in disease eradication: the larger they are, the larger the disease reduction. Conditions for the Hopf bifurcation at the coexistence equilibrium are instead more stringent.

The behavior of the model introduced in [67], with quadratic population interactions and standard disease incidence, is similar: the origin is destabilized by a low enough disease contact rate and the 
healthy-prey-only and the predator-free equilibria can be attained, while in [114] this does not happen as they are unconditionally unstable.

\subsection{The Holling-Tanner underlying demographics}

Assuming that predators gain the same from feeding on sound and sick prey predation, so that the disease has no negative consequences, a model with this dynamics in nondimensional form is, [68]:

$$
S^{\prime}=S(1-S)-\frac{S Z}{a+S+I}-\lambda S I, \quad I^{\prime}=\lambda S I-\frac{\eta I Z}{a+S+I}-\gamma I, \quad Z^{\prime}=Z\left[\delta-\frac{\beta Z}{S+I}\right] .
$$

Boundedness of the trajectories is shown if $4 \beta \gamma>\delta^{2}$, the origin and the predator-free equilibria are unconditionally unstable. The stability of the disease-free and of the interior equilibria can be characterized. The equilibria of the purely demographic model are also equilibria of the ecoepidemic model, with the same population levels. From the second equation, if $\lambda<\gamma$ the infection will not spread, noting that from the first equation $\lim _{t \rightarrow \infty} \sup S \leq 1$. Local stability of the disease-free point $E_{2}=\left(S_{2}, 0, Z_{2}\right)$ ensures also its global stability. At this equilibrium, if $\eta \geq 1$ and $\delta S_{2} \leq \beta\left(a+S_{2}\right)^{2}$, when $\lambda$ crosses the critical values $\lambda_{h \pm}=\frac{1}{2}\left[\psi_{1} \pm \sqrt{\psi_{1}^{2}-4 \psi_{2}}\right]$ where $\psi_{2}=\chi_{2}+\delta S_{2}^{-2}$ and

$$
\begin{aligned}
\psi_{1} & =\frac{Z_{2}}{a+S_{2}} \frac{2 \eta\left(a+S_{2}\right)-S_{2}}{S_{2}\left(a+S_{2}\right)}+1+\frac{\gamma+\delta}{S_{2}}, \\
\chi_{2} & =\left[\frac{\eta Z_{2}}{S_{2}\left(a+S_{2}\right)}+\frac{\gamma}{S_{2}}\right]\left[\frac{\eta Z_{2}}{S_{2}\left(a+S_{2}\right)}+\frac{\delta}{S_{2}}+1-\frac{Z_{2}}{\left(a+S_{2}\right)^{2}}\right]+\frac{\delta}{S_{2}^{2}},
\end{aligned}
$$

the system undergoes a Hopf-bifurcation. Letting $\beta_{h}=\delta S_{2}^{2}\left(a+S_{2}\right)^{-2}\left(\delta+S_{2}\right)^{-1}$, when $\delta S_{2}=\beta\left(a+S_{2}\right)^{2}$ or $\beta=\beta_{h}$, the system again experiences another Hopf-bifurcation at $\beta=\beta_{h}$. Furthermore, at $\lambda=$ $\lambda^{[2]}=S_{2}^{-1}\left(\eta Z_{2}\left(a+S_{2}\right)^{-1}+\gamma\right)$ the system exhibits a transcritical bifurcation. The local stability of the coexistence equilibrium can further be characterized; it implies also its global stability if $\lambda<\lambda_{\min } \equiv$ $\eta \delta \beta^{-1}$.

Note that here too, the stability analysis for the disease-free equilibrium $E_{2}$ differs substantially from the corresponding point in the purely demographic model, requiring the additional condition $\lambda<\lambda^{[2]}$. A disease with a suitably large value of the contact rate destabilizes the purely demographic coexistence equilibrium. Thus once again the infection is seen to play a substantial influence on the ecosystem.

The corresponding model with disease in predators, already in rescaled form, is considered [69]:

$$
N^{\prime}=N\left(r-H N-\frac{P}{1+N}-\frac{p Y}{1+N}\right), \quad P^{\prime}=P\left(\frac{N}{1+N}-m Y+n\right), \quad Y^{\prime}=Y\left(m P+\frac{p N}{1+N}-d\right),
$$

where predators have other food sources available. The total predator population size at the coexistence equilibrium $E_{*}$ is $Z_{*} \equiv P_{*}+Y_{*}$ with $P_{*}=\left[d+(d-p) N_{*}\right]\left[m\left(1+N_{*}\right)\right]^{-1}$ and $Y_{*}=\left[(n+1) N_{*}+n\right][m(1+$ $\left.\left.N_{*}\right)\right]^{-1}$. Differentiating with respect to the prey size, we respectively find:

$$
\frac{d P_{*}}{d N_{*}}=\frac{-p}{m\left(1+N_{*}\right)^{2}} \leq 0, \quad \frac{d Y_{*}}{d N_{*}}=\frac{1}{m\left(1+N_{*}\right)^{2}} \geq 0, \quad \frac{d Z_{*}}{d N_{*}}=\frac{1-p}{m\left(1+N_{*}\right)^{2}} .
$$

Thus an increase of the prey population size decreases the healthy predators population, while it increases the size of the infected predators. Now $p<1$ because infected predators are less active than the healthy ones, so that the last derivative is positive. Hence an increase of the prey population produces also an increase in the total predator abundance, but this occurs through an increase of the disease prevalence.

\subsection{Ratio-dependent models}

Ratio-dependent models contain a response function, [2], that depends on the relative size of the predator and prey populations, i.e.:

$$
\frac{N}{\frac{N}{Z}+1}=\frac{N Z}{N+Z}
$$


In case of ecoepidemics, we need to account for the splitting of the predators population among healthy and infectious individuals, $Z=P+Y$. Consider the system:

$$
\begin{aligned}
& N^{\prime}=N\left[r-B N-m \frac{P-p Y}{Y+P+N}\right], \quad P^{\prime}=P\left[\frac{e m N}{Y+P+N}-\lambda Y+\mu\right], \\
& Y^{\prime}=Y\left[\lambda P+\frac{e m p N}{Y+P+N}-d\right] .
\end{aligned}
$$

Here the predation rate $m$ is reduced by a factor $p<1$ for the infected predators, $q$ is the natural death rate, $b$ the birth rate, $\delta$ the disease related mortality, $B^{-1}$ the prey carrying capacity, $e<1$ the food conversion rate and where $d=q+\delta$ and $\mu=b-q \in \mathbf{R},[70]$.

When $\mu<0$, if also $e m<\max \left\{-\mu, d p^{-1}\right\}$, the whole predator population disappears and with it the disease. The origin can be attained by the system's trajectories, the biological reason being that the infected predate at a smaller rate than the healthy predators. Thus a heavily infected predator may cause total collapse of the ecosystem.

In this model the disease prevents predators disappearance. Their extinction can instead occur in the purely demographic model. When the prey have unlimited food supply the disappearance of only the predators cannot occur. Here the healthy predators-free equilibrium does not exist, contrary to what happens in [69], so that infected predators and prey cannot persist in the absence of susceptible predators, when the predation involves a substantial effort. The disease in the predators may prevent ecosystem collapse. Combining these remarks with the findings of [68], the presence of a disease thus in either population can destabilize an otherwise stable equilibrium and therefore act as a biological control.

\subsection{Structured models}

So far we have looked at populations as a whole. In this Section we instead distinguish between sets of individuals within a single population, classifying them according to some specific feature. It is well known that in demographic models the populations can be structured by looking at age cohorts, for instance, or to the size of their individuals. These distinctions could be applied also to ecoepidemic models, but they are not the only possibility. We present here two related situations, using these ideas instead in the context of the stage of the disease, rather than on the biological age of the individuals.

At first, the following model can be formulated, [133], where the infected prey and predator populations are represented by the densities $i \equiv i(t, \sigma), y \equiv y(t, \sigma)$, where $\sigma$ is the stage of the disease. Consider the following system of equations:

$$
\begin{aligned}
& S^{\prime}=S(a-b P-e S)-e S I-S \int_{0}^{\infty} \alpha(\sigma) i(t, \sigma) d \sigma, \quad \frac{\partial i}{\partial t}+\frac{\partial i}{\partial \sigma}=i[-e(S+I)-m P-p Y], \\
& P^{\prime}=P(-c+d S)+g P I-P \int_{0}^{\infty}[\beta(\sigma) y(t, \sigma)+\gamma(\sigma) i(t, \sigma)] d \sigma, \quad \frac{\partial y}{\partial t}+\frac{\partial y}{\partial \sigma}=y[-h+n I],
\end{aligned}
$$

where the last term in the last equation represents the benefit that infected predators have by hunting infected prey. The total infected prey and predators are obtained summing over the whole set of disease stages:

$$
I(t)=\int_{0}^{\infty} i(t, \sigma) d \sigma, \quad Y(t)=\int_{0}^{\infty} y(t, \sigma) d \sigma .
$$

Initial conditions containing the starting distribution of the disease stages complement the system. This formulation allows then to model the fact that the disease transmission depends on the state of the disease itself, via the transmissibilities $\alpha$ among prey, $\beta$ among predators and $\gamma$ from prey to predators. This is well known for instance in human diseases. For measles an individual is infectious only in the very first days after contracting the disease, and well in advance of showing any symptom of it. 
In the context of nonlinear partial differential equations, the well-posedness of the problem is a fundamental issue. Following the technique of the classical models introduced long ago, [58], it is possible to show existence and uniqueness results also for (4.9).

Another very interesting model is presented in [85] for the aquatic environment. Let $B(t)$ the free bacteria population in the water, which is not an environment that can sustain them. They can infect healthy fish $S(t)$, within which they reproduce. The infected fish density $i(b, t)$ depends on $b$, the state of the disease, expressed by the amount of bacteria within the hosts. The model is:

$$
\begin{aligned}
& S^{\prime}(t)=r S(t)(K-S(t))-S(t) f(B(t)), \quad B^{\prime}(t)=\mu B(t)+\int_{b_{0}}^{\bar{b}} b \nu(b) i(b, t) d b, \\
& \frac{\partial i(b, t)}{\partial t}+\frac{\partial \beta b i(b, t)}{\partial b}=-\nu(b) i(b, t), \quad \beta b_{0} i\left(b_{0}, t\right)=S(t) f(B(t)), \quad b^{\prime}(t)=\beta b(t) .
\end{aligned}
$$

The last equation models the bacteria reproduction, which occurs only within the hosts, via a Malthus law with birth rate $\beta$. In the above expressions, it is assumed that the disease manifests itself whenever its state is higher than $b_{0}$, while no fish survives past the stage $\bar{b}$. Infected fish cannot reproduce. The term $f(B(t)) d t$ represents the ratio of new fish infections to the susceptible fish during the time interval $(t, t+d t)$. Initial conditions are $i(b, 0)=i_{0}(b), S(0)=S_{0}, B(0)=B_{0}, b(0)=b_{0}$. Fish mortality $\nu$ depends on the disease stage; note that bacteria contained in the dead infected fish are recycled in the system, via the integral term in the $B$ equation; $f(B(t)) d t$ represents the ratio of new infected fish in the time interval $(t, t+d t)$ over the healthy fish population.

Solving along the characteristics in the $b-t$ space, the PDE system can be turned into the distributed delay ODE system:

$$
\begin{aligned}
& B^{\prime}(t)=-\mu B(t)+\int_{b_{0}}^{\bar{b}} b \nu(b) \pi(b) g(t, b) d b, \quad S^{\prime}(t)=r S(t)(K-S(t))-S(t) f(B(t)), \\
& g(t, b)=\frac{1}{\beta b_{0}} S\left(t-\frac{1}{\beta} \ln \left(\frac{b}{b_{0}}\right)\right), \quad \pi(b)=\exp \left(-\int_{0}^{\frac{1}{\beta} \ln \left(\frac{b}{b_{0}}\right)}\left(\beta+\nu\left(b_{0} \exp (\beta \sigma)\right)\right) d \sigma\right) .
\end{aligned}
$$

If instead the mortality is concentrated in a neigborhood of the point $\bar{b}$, a model with discrete delays is obtained:

$$
B^{\prime}(t)=-\mu B(t)+\bar{b} S(t-\tau) f(B(t-\tau)), \quad S^{\prime}(t)=r S(t)(K-S(t))-S(t) f(B(t)),
$$

with $S_{0}=\varphi_{1}>0, B_{0}=\varphi_{2}>0, \tau=\beta^{-1} \ln \left(\bar{b} b_{0}^{-1}\right)$. Assuming in this case a Holling type II response function, $f(x)=x\left(K_{1}+x\right)^{-1}$, the well posedness of the problem, positivity and boundedness of the solutions are established. The origin is an unstable equilibrium, the threshold quantity $R=\bar{b} K\left(\mu K_{1}\right)^{-1}$ regulates the system's outcome: for $R<1$ the environment becomes disease-free, conversely the bacteria survive endemically in the ecosystem, for $R=1$ there is a transcritical bifurcation for which the endemic equilibrium emanates from the disease-free one. Conditions for Hopf bifurcations are investigated analytically and numerically.

A simplification of the age-structured model studied in [5] is the following one, [48]:

$$
\begin{aligned}
& \frac{\partial i}{\partial t}+\frac{\partial i}{\partial a}+\mu_{1}(a, N(t)) i(a, t)=K(a) I(t) s(a, t)-M_{1} i(a, t) Z(t), \\
& \frac{\partial s}{\partial t}+\frac{\partial s}{\partial a}+\mu_{2}(a, N(t)) s(a, t)=-K(a) I(t) s(a, t)-M_{2} s(a, t) Z(t), \\
& Z^{\prime}=m Z-n Z^{2}+\varepsilon M_{1} I(t) Z(t)+\varepsilon M_{2} S(t) Z(t), \quad i(a, 0)=i_{0}(a), \quad s(a, 0)=s_{0}(a), \quad Z(0)=Z_{0}, \\
& i(0, t)=q \int_{0}^{\infty} \beta(a, N(t)) i(a, t) d a, \quad s(0, t)=q \int_{0}^{\infty} \beta(a, N(t))[s(a, t)(1-q) i(a, t)] d a,
\end{aligned}
$$


with $i$ being the density of infected prey, $s$ the one of the healthy prey and

$$
I(t)=\int_{0}^{\infty} i(a, t) d a, \quad S(t)=\int_{0}^{\infty} s(a, t) d a, \quad N=S+I .
$$

Global stability results are derived in the corresponding model in [48] with age-independent coefficients:

$$
\begin{aligned}
& I^{\prime}=q\left(\beta(N) I-\mu_{1}(N) I+K I S-m_{1} I Z\right. \\
& S^{\prime}=\beta(N) S[S+(1-q) I]-\mu_{2}(N) S-K I S-M_{2} S Z, \quad Z^{\prime}=m Z-n Z^{2}+\varepsilon M_{1} I Z+\varepsilon M_{2} S Z
\end{aligned}
$$

\section{More actors}

In the ecoepidemic system, the possibility of the presence of multiple populations of the same type, but differing for some characteristics, can be considered in several settings. We can have diseases affecting both hosts at the same time; in this case we briefly look also at possible control measures. Another possibility is to have several food sources available for predators, so that if other alternative prey are available, they would turn to a different diet; another one is the simultaneous occurrence of different strains of infections; a further situation considers instead populations at different trophic levels, i.e. food chains. The situations in which the ecosystem may be split among several interconnected environments can also be considered in the context of ecoepidemics. Finally, for an SIRS model, chaotic behavior is shown.

\subsection{Epidemics in both populations}

We now outline a few models for which both populations can get infected, to illustrate some of the several possibilities that can occur.

In [60], the mechanism for which the epidemics can remain in the environment passing back and forth from one population to the other one is illustrated. In this case the specific feature of the model is the absence of a direct transmission between infected individuals and susceptibles of the same population. Indeed, the disease is assumed to be caused by parasites released in the environment by the infected predators $Y$ and possibly assumed by the healthy prey $S$. The susceptible predators $P$ get the infection by feeding on infected prey $I$. This is expressed by the last term of the last equation in (5.1). The underlying demographic is a Rosenzweig predator-prey system:

$$
\begin{aligned}
& S^{\prime}=a N-\frac{a S N}{K}-\frac{S}{A+S+\rho I} Z-\beta S Y, \quad I^{\prime}=\beta S Y-\frac{a I N}{K}-\frac{\rho I}{A+S+\rho I} Z, \\
& P^{\prime}=-\frac{c B}{A+B} P+c \frac{S+\rho I}{A+S+\rho I} Z-\kappa \frac{\rho I}{A+S+\rho I} P, \quad Y^{\prime}=-\frac{c B}{A+B} P+\kappa \frac{\rho I}{A+S+\rho I} P .
\end{aligned}
$$

Epidemic thresholds are derived, exploiting the fact that the Jacobian is a partitioned matrix. This parasite may be nonpersistent, if the predators cannot survive just on the totally infected prey, leaving only the healthy prey thriving in the environment. But when predators are sufficiently numerous and transmission rates are large enough coexistence becomes possible if the diet based only on infected prey is harmless. The presence of the parasites can remain endemic if the product of the trasmission rates exceeds a certain threshold.

The model presented in [82] contains essentially two different diseases, as there is no transmission among the two populations. Here, new infected are produced by contact of susceptibles with infected of their own species. Modelling the process with simple mass action transmission, we have:

$$
\begin{aligned}
& S^{\prime}=r S\left(1-\frac{N}{K}\right)-\lambda_{1} I S-\frac{a_{1} S}{1+a_{1} S} Z, \quad I^{\prime}=\lambda_{1} I S-\frac{a_{2} I}{1+a_{2} I} Z-\mu I, \\
& P^{\prime}=\frac{\beta S P}{1+a_{1} S}-\gamma_{1} P-\frac{\lambda_{2} I P}{1+a_{2} I}, \quad Y^{\prime}=\frac{\lambda_{2} I P}{1+a_{2} I}-\gamma_{2} Y .
\end{aligned}
$$


In the demographic model the threshold $R_{1}$ is identified, for which either the predator-free equilibrium, for $R_{1}<1$, or coesistence, for $R_{1}>1$, are globally asymptotically stable, or coexistence occurs through limit cycles when $R_{1}>1+\beta\left[\alpha_{1} \gamma_{1}\left(1+a_{1} K\right)\right]^{-1} \equiv 1+H_{*}$. In the ecoepidemic model, additional thresholds arise, namely:

$$
R_{1}=\frac{\beta K}{\gamma_{1}\left(1+a_{1} K\right)}, \quad R_{0}=\frac{K \lambda_{1}}{\mu}, \quad \bar{R}_{1}=\frac{\beta \bar{S}}{\gamma_{1}\left(1+a_{1} \bar{S}\right)}, \quad R_{0}^{*}=\frac{\lambda_{1} S^{*}}{\alpha_{2} P^{*}+\mu}, \quad \widetilde{R}_{1}=\frac{\beta \widetilde{S}}{\gamma_{1}\left(1+a_{1} \widetilde{S}\right)}
$$

where $E_{B}=(K, 0)$ is the predator-free equilibrium in the disease-free system, $\bar{E}_{B}=(\bar{S}, \bar{I}, 0,0)$ represents the predator-free environment, with endemic disease in the prey, $E^{*}=\left(S^{*}, 0, P^{*}, 0\right)$ is the disease-free equilibrium and $\widetilde{E}=(\widetilde{S}, \widetilde{I}, \widetilde{P}, \widetilde{Y})$ the coexistence one. Let us further write $H^{*} \equiv \lambda_{2} \bar{I}\left[\gamma_{1}\left(1+a_{2} \bar{I}\right)\right]^{-1}$. The model dynamics can be completely described by all these thresholds. Each equilibrium is locally asymptotically stable in the following cases:

- $E_{B}$ is for $R_{0}<1$ and $R_{1}<1$;

- $\bar{E}_{B}$ requires either $R_{0}>1$ and $R_{1} \leq 1$, or $R_{0}>R_{0}^{*}>1, \bar{R}_{1}<1+H^{*}$ and $1<R_{1}<1+H_{*}$, or $R_{0}>1>R_{0}^{*}, \bar{R}_{1}<1+H^{*}$ and $1<R_{1}<1+H_{*}$, or finally $R_{0}>1>R_{0}^{*}, \bar{R}_{1}<1+H^{*}$ and $R_{1}>1+H_{*}$. Note that in these last two cases $\widetilde{E}$ is numerically shown to be unstable.

- $E_{B}^{*}$ needs instead alternatively either $R_{0} \leq 1$ and $1<R_{1}<1+H_{*}$, or else $1+H_{*}>R_{1}>\bar{R}_{1}>1+H^{*}$ and $R_{0}>1>R_{0}^{*}$, or finally $R_{0}>1>R_{0}^{*}, \bar{R}_{1}<1+H^{*}$ and $1<R_{1}<1+H_{*}$.

- Further, in the $S P$ plane limit cycles have been found whenever either $R_{0}^{*}<R_{0} \leq 1$ and $R_{1}>1+H_{*}$, or else $R_{0}>1>R^{*}, \bar{R}_{1}>1+H^{*}$ and $R_{1}>1+H_{*}$, or finally $R_{0}>1>R_{0}^{*}, \bar{R}_{1}<1+H^{*}$ and $R_{1}>1+H_{*}$.

In [61] instead both SIS and SIR models are discussed in the ecoepidemic setting. The basic demographic is the predator-prey model with prey logistic growth and quadratic interactions, which has an attractive coexistence equilibrium. The infection process is modeled both via mass action and standard incidence. The predators can get diseased also when hunting infected prey. Thresholds are derived to ensure either disease eradication or that instead it persists in the predators, if their hunting rate is high enough so that they can thrive. Global stability results are also obtained.

In [112] epidemics-affected competing models are discussed, with the disease modeled via the standard incidence and affecting both populations $N_{1}$ and $N_{2}$, with $I_{1}$ and $I_{2}$ denoting the two infected classes:

$$
\begin{aligned}
& N_{1}^{\prime}=N_{1}\left[r_{1}\left(1-\frac{N_{1}+\alpha_{12} N_{2}}{K_{1}}\right)-\varepsilon_{1} I_{1}+f_{1} \chi_{1} r_{1} I_{1} \frac{N_{1}+\alpha_{12} N_{2}}{K_{1}}\right], \\
& I_{1}^{\prime}=\left(\beta_{11} I_{1}+\beta_{12} I_{2}\right)\left(1-I_{1}\right)-I_{1}\left[d_{1}-\varepsilon_{1} I_{1} \chi_{1} r_{1}\left(1-f_{1} I_{1}\right) \frac{N_{1}+\alpha_{12} N_{2}}{K_{1}}\right], \\
& N_{2}^{\prime}=N_{2}\left[r_{2}\left(1-\frac{N_{2}+\alpha_{21} N_{1}}{K_{2}}\right)-\varepsilon_{2} I_{2}+f_{2} \chi_{2} r_{2} I_{2} \frac{N_{2}+\alpha_{21} N_{1}}{K_{2}}\right], \\
& I_{2}^{\prime}=\left(\beta_{22} I_{2}+\beta_{21} I_{1}\right)\left(1-I_{2}\right)-I_{2}\left[d_{2}-\varepsilon_{2} I_{2} \chi_{2} r_{2}\left(1-f_{2} I_{2}\right) \frac{N_{2}+\alpha_{21} N_{1}}{K_{2}}\right] .
\end{aligned}
$$

For size-independent birth rates the system simplifies a bit, $\chi_{i}=0$, and uncouples as the equations for infectives become independent of the other ones. The standard results on the system's outcomes are obtained: when the disease dies out, either competitive exclusion, or else stable coexistence, occurs. But the disease affects the competition outcomes, for instance it can weaken a superior competitor so that instead coexistence becomes possible. The disease can also remain endemic in both populations, but it could also drive one population to extinction. The type of disease contact rate possibly changes the ultimate behavior of the competing system. The paper extends also to consider SIRS models. 
On the same line of two different infections, the system in [45] allows instead vertical disease transmission and once again the infections in the two populations are independent of each other:

$$
\begin{aligned}
& S^{\prime}=r_{1} S\left(1-\frac{S}{K}\right)-\lambda_{1} I S-a S Z, \quad I^{\prime}=r_{2} I\left(1-\frac{X}{K}\right)+\lambda_{1} I S-\beta I Z-m I, \\
& P^{\prime}=e_{1} \alpha S P+e_{2} \beta I P-\lambda_{2} P Y-\mu P, \quad Y^{\prime}=\lambda_{2} P Y+e_{3} \alpha S Y+e_{4} \beta I Y+\delta Y .
\end{aligned}
$$

In this case twenty reproduction numbers are identified to appropriately characterize the system's behavior.

Finally, as for (5.1), in [38] the model allows the disease to cross the species barrier in both ways. Namely, a healthy prey that survives the interaction with an infected predator could catch the disease. On the other hand, a healthy predator can become infected by ingesting a diseased prey, but also e.g. through ground contaminated by the infected prey. Among the findings, the healthy predators establish themselves in the disease-free ecosystem if three conditions hold. They need to exert hunting on healthy prey at a high rate, or alternatively, the predators' natural mortality is low; further, if the predators' disease-related mortality compared with the natural one is sufficiently high; finally, if the healthy predators hunt the susceptible prey at a high rate or the disease is transmitted from the infected predators to the prey at a low rate. The predator-free environment is feasible if the sound prey reproduce quicker than the infected ones and the prey intraspecific population pressure lies in a certain interval. It is stable if the following two conditions hold. First, the ratio of disease-related and natural predators' mortalities must exceed a critical value. Secondly, the ratio between the following two quantities must be bounded above. These quantities are a demographic one, the conversion rate of infected prey into newborn healthy predators, and an epidemiological one, the disease transmission rate among prey. These conditions are required also for the disease to become a pandemic, i.e. affecting both populations and wiping out all the susceptibles in the system. But in addition, two other requirements are needed. The predators' conversion rate of infected prey into healthy newborns is smaller than the disease transmission rate among the predators. Then the net reproduction rate of healthy prey must be bounded above.

Simulations reveal the following results. Coexistence of all the four subpopulations occurs for low values of the prey intraspecific disease transmission, when it is larger the healthy prey disappear. A similar behavior occurs also for the interspecific disease transmission rate from infected predators to healthy prey. High values of the opposite contact rate, from infected prey to healthy predators, lead instead to a pandemic, in which only infected populations survive. A low predators intraspecific disease contact rate renders the system disease-free, while again for higher values coexistence is observed, then first the healthy prey get extinguished, and finally also the healthy predators.

\subsection{Harvesting and control}

We briefly mention here the fact that populations can be exploited as renewable resources also in the context of ecoepidemics.

The model in [40] extends the one of [38] to account additionally for the harvesting of the populations at possibly different rates, $h, \ell, \pi$ and $n$, and reads:

$$
\begin{aligned}
& S^{\prime}=S\left[r_{1}-\frac{N}{K}-\gamma I-\left(\beta+b_{3}\right) Y-b_{1} P-h\right], \quad I^{\prime}=I\left[r_{2}-\nu+\gamma S-\frac{N}{K}-b_{2} P-b_{4} Y-\ell\right]+\beta S Y, \\
& P^{\prime}=P\left[-m-\alpha I-\eta Y+e\left(b_{1} S+b_{2} I\right)-\pi\right], \quad Y^{\prime}=Y\left[-m-\mu+\eta P+e\left(b_{3} S+b_{4} I\right)-n\right]+\alpha I P .
\end{aligned}
$$

The possible effects of harvesting on the system's equilibria of each of the subpopulations are discussed. In the absence of harvesting, [38], the healthy prey-only equilibrium is stable if either the intraspecific competition is high enough, or the disease transmission rate is low. Instead, in [40] in presence of harvesting, the prey become disease-free even if these conditions are not met. Healthy predators harvesting leads always to endemic coexistence in both populations. Harvesting of infected prey at low levels produces coexistence, for larger values the prey become disease-free. Harvesting makes the system disease-free also 
if the prey interspecific contact rate is low. But in its absence, the system would attain coexistence, in view of the disease spreading from infected prey to predators. Harvesting, however, does not affect the disease intraspecific spread among the predators. Finally, a high infected prey predation rate removes the disease from the prey; but, in these conditions, harvesting makes the system disease-free.

Harvesting in a predator-prey-parasite model is also considered in [9], showing that, using impulsive controls in both healthy and diseased prey, it is possible to drive the sustained oscillations of the system so that the disease-free equilibrium is achieved.

This issue becomes important when one of the populations in consideration is considered a nuisance. Control measures apt to keep it in check are considered in [84]. The following ecoepidemic model with infected prey is considered in which a control, represented by indiscriminate culling of all the populations, is employed:

$$
\begin{array}{r}
S^{\prime}=r S\left(1-\frac{S+\eta I}{K}\right)-\alpha S I-\beta \frac{S Z}{a+S}-\varepsilon_{1} u S, \quad I^{\prime}=I(\alpha S-b Z-\sigma I)-\varepsilon_{2} u I, \\
Z^{\prime}=\ell \beta \frac{S Z}{a+S}+(m-n) b I Z+g Z\left(1-\frac{S+\eta I}{K}\right)-\varepsilon_{3} u Z,
\end{array}
$$

where the infected pests are food for the predators and therefore contribute to their reproduction at rate $m$, but also can harm them, causing an extra mortality rate $n$. Note also that the predators have additional food sources. The optimal control strategy to minimize infestants impact is assessed.

In this context, we mention also the investigations [88], with an SIRV model for a disease, including a class of vaccinated individuals, and [87], in which in addition the susceptible and infected vectors are introduced.

In a different situation, looking at agroecosystems without explicitly building a control problem, we mention also the use of spiders for fighting pests in fruit orchards and vineyards, [31,139].

Since the predator-prey dynamics can intimately be related to the ones of pests, the biological control of the latter can also be obtained by transmissible diseases. On the other hand, some diseases are also related to vectors, see especially [15]. In it the disease-affected prey are considered together with their predators and the disease vectors $V$ :

$$
\begin{array}{r}
S^{\prime}=r S\left(1-\frac{S+I}{K}\right)-\lambda S V, \quad I^{\prime}=\lambda S V-\frac{\Pi Z}{\Gamma+I}-\xi I, \\
Z^{\prime}=\frac{C_{P} \Pi Z}{\Gamma+I}-d_{Z} Z-\varepsilon_{Z} Z^{2}, \quad V^{\prime}=\kappa \xi I-\mu_{V} V .
\end{array}
$$

The systems attains limit cycles, the stability of which is investigated.

In this respect, the biological control can be obtained also by using parasitoids of the pests. This is an interesting issue, as these models are very closely related to ecoepidemic ones, see e.g. [100], in which the following model is introduced

$$
S^{\prime}=r S\left(1-\frac{S}{K}\right)-n_{1} S-\beta S Z, \quad I^{\prime}=\beta S Z-m_{2} I-n_{2} I, \quad Z^{\prime}=\gamma n_{2} I-m_{3} Z+U,
$$

where $S$ denotes the healthy sugarcane borer larvae, $I$ are the larvae that are parasitized, $Z$ are the parasitoids and $U$ is the control, implemented via parasitoids release in the environment. The parameters $m_{i}, i=2,3$, are mortality rates of parasitized larvae and parasitoids respectively, while $n_{1}$ denotes the moulting of larvae into pupae and $n_{2}$ represents the fraction of parasitized larvae from which adult parasitoids emerge. The goal is to keep the sugarcane borer larvae at a low level, below economic injury, by suitably and efficiently releasing the parasitoids. A nonlinear control is instead introduced in [99].

\subsection{Prey switching}

The possibility of having more than one prey available allows possibly the predators to change from time to time the type of resource, according to their taste or the ease with which to catch it, $[89,90,104]$. In 
[81] one such model is presented in the context of ecoepidemiology. In contrast to purely demographic models, in which the feeding switching occurs among two different prey populations, see e.g. [127], here the predators hunt healthy and diseased prey. The model is:

$$
\begin{aligned}
& S^{\prime}=\left[r_{1}\left(1-\frac{S+I}{K}\right)-\gamma I-\frac{a S Z}{S+I}\right] S+\delta I, \quad I^{\prime}=\left[r_{2}\left(1-\frac{S+I}{K}\right)+\gamma S-\delta-\frac{|b| I Z}{S+I}\right] I, \\
& Z^{\prime}=\left[-r_{3}+\frac{a S^{2}}{S+I}+\frac{b I^{2}}{S+I}\right] Z,
\end{aligned}
$$

accounting for, via the $|b|$ terms, both the case for which for the predators the infected prey is respectively harmless, $b>0$, or toxic, $b<0$. With obvious notations, the logistic reproduction occurs at different rates for both healthy and infected prey, i.e. the disease is vertically transmitted. The disease dynamics is contained in the parameters $\gamma$ and $\delta$ denoting respectively the contact and recovery rates. The last terms in each equation represent the switching mechanism and are mathematically characterized by a term in the numerator of overall degree 3 . The switching interpretation appears clearly by observing that

$$
\frac{a S Z}{S+I}=\frac{a Z}{1+\frac{I}{S}}
$$

for which whenever the size of the infected population becomes larger than the size of the healthy one, the denominator increases, and therefore the fraction has a smaller influence in the first equation dynamics. The opposite phenomenon simultaneously occurs in the second equation, so that more infected are removed by predators' hunting than healthy prey. The converse effect is obtained for a smaller size of the infected population, compared with the size of the healthy one.

In the model the disease basic reproduction number $\mathcal{R}_{0}=\gamma K \delta^{-1}$ and the predators' invasion number $\mathcal{R}_{1}=a K r_{3}^{-1}$ play an essential role in identifying the systems' equilibria stability, in particular showing that the disease-free equilibrium emanates from the prey-only one whenever $\mathcal{R}_{1} \geq 1$. In addition, the disease can be eradicated whenever $\mathcal{R}_{0}$ is suitably bounded from above in terms of $\mathcal{R}_{1}$. Hopf bifurcations are shown to occur.

\subsection{Two strains}

The influence of several diseases on a single population is a topic recently considered in epidemiology. The corresponding ecoepidemiological situation has been considered in [22] for the symbiotic case and in the very recent paper [27] for the competing demographic system. Still indicating with $N, S, I$ and $Z$, $P, Y$ the two populations and denoting respectively by $J$ and $W$ their second diseases, these models are:

$$
\begin{aligned}
& S^{\prime}=-n S-\lambda J S-\beta I S+a S Z+\xi J+\varphi I, \quad J^{\prime}=\lambda J S-\mu J-\xi J+q J Z, \\
& I^{\prime}=\beta I S-\nu I-\varphi I+r I Z, \quad Z^{\prime}=-m Z+e S Z+f J Z+g I Z
\end{aligned}
$$

for the symbiotic case and for the competing one

$$
\begin{aligned}
& N^{\prime}=s\left(1-\frac{N}{L}\right) N-a N P, \quad P^{\prime}=r\left(1-\frac{P}{K}\right) P-b P N-\lambda Y P-\beta W P+\psi Y+\varphi W, \\
& Y^{\prime}=(\lambda-p) Y N-\psi Y-\mu Y-e N Y, \quad W^{\prime}=(\beta-q) W P-\varphi W-\nu W-f N W .
\end{aligned}
$$

The major finding in the competition case (5.10) is the discovery that the two diseases cannot be both simultaneously supported by the environment, under the assumptions made. In fact, the analysis of the equilibria in the remaining subsystems, in which only the strongest disease is present, reduces to two copies of the one performed for the model already studied in [131], and shows also sustained populations oscillations. In [27] the latter are thoroughly investigated in the two-parameter subspaces. 
The feasibility and stability of equilibria are discussed in terms of "reduced" disease-free competitor recruitment rate $\mathcal{R}_{N}$ and the corresponding one for the disease-affected one, $\mathcal{R}_{P}$, and the "reduced loss rates" for the two strains, $\mathcal{L}_{Y}$ and $\mathcal{L}_{W}$. These quantities are defined as follows:

$$
\mathcal{R}_{N}:=\frac{s}{a}, \quad \mathcal{R}_{P}:=\frac{r}{b}, \quad \mathcal{L}_{Y}:=\frac{\psi+\mu}{\lambda-p}, \quad \mathcal{L}_{W}:=\frac{\varphi+\nu}{\beta-q} .
$$

The disease-free equilibria are either the survival of just the disease-unaffected population $N$, at carrying capacity $L$, stably achieved if and only if this carrying capacity is large enough, $L>\mathcal{R}_{N}$, or by only the susceptibles $P$ of the disease-affected population, stable for the carrying capacity $K$ lying in the suitable window $\mathcal{R}_{N}<K<\min \left\{\mathcal{L}_{Y}, \mathcal{L}_{W}\right\}$. Coexistence in a disease-free environment is feasible for $K<\mathcal{R}_{N}$ and $L<\mathcal{R}_{P}$, stable if

$$
\begin{aligned}
& K \mathcal{R}_{\mathcal{N}}\left(L-\mathcal{R}_{\mathcal{P}}\right) \\
& <\min \left\{\mathcal{L}_{\mathcal{W}}\left(K L-\mathcal{R}_{\mathcal{N}} \mathcal{R}_{\mathcal{P}}\right)+\frac{f L}{\beta-q} \mathcal{R}_{\mathcal{P}}\left(K-\mathcal{R}_{\mathcal{N}}\right), \mathcal{L}_{\mathcal{Y}}\left(K L-\mathcal{R}_{\mathcal{N}} \mathcal{R}_{\mathcal{P}}\right)+\frac{e L}{\lambda-p} \mathcal{R}_{\mathcal{P}}\left(K-\mathcal{R}_{\mathcal{N}}\right)\right\}
\end{aligned}
$$

The one-strain endemic equilibrium is feasible if $K \geq \mathcal{L}_{Y}$ and stable if $\mathcal{R}_{N}<\mathcal{L}_{Y}<\mathcal{L}_{W}$. The coexistence of one strain and the healthy populations is feasible for $\mathcal{R}_{N} \geq \mathcal{L}_{Y}, P_{6}>\psi \lambda^{-1}, r P_{6}+b K N_{6} \leq r K$; stability conditions are rather complicated.

\subsection{Food chains}

In [47] a simple three level food web subject to a disease affecting the bottom prey is considered. The top predators $Z$ feed just on the intermediate population $V$, the bottom prey $N=S+I$ grows logistically and is affected by a recoverable disease that is not vertically transmitted:

$$
\begin{aligned}
& Z^{\prime}=-m Z+p V Z, \quad V^{\prime}=-\ell V+e S V-h V Z+q I V, \\
& I^{\prime}=\beta I S-n I V-\gamma I-\nu I, \quad S^{\prime}=a S\left(1-\frac{S+I}{K}\right)-c V S-\beta S I+\gamma I .
\end{aligned}
$$

In the absence of the disease the bottom-prey-only equilibrium $Q_{1}=(0,0, K)$, the top-predator-free equilibrium $\widetilde{Q}=(0, \widetilde{V}, \widetilde{N})$, and the coexistence equilibrium $Q^{*}=\left(Z^{*}, V^{*}, N^{*}\right)$ are shown to be globally asymptotically stable, using respectively the Lyapunov functions:

$$
\begin{aligned}
& L_{1}=\frac{c h}{e p} Z+\frac{c}{e} V+\left(N-K \ln \frac{N}{K}\right), \\
& \widetilde{L}=-\frac{c h}{e} V(Z-\widetilde{Z})+\frac{c}{e}[e N-\widetilde{N}-h(Z-\widetilde{Z})] V+\left[a\left(1-\frac{N}{K}\right)-c V\right](N-\widetilde{N}), \\
& L^{*}=\frac{c h}{e p}\left(Z-Z^{*} \ln \frac{Z}{Z^{*}}\right)+\frac{c}{e}\left(V-V^{*} \ln \frac{V}{V^{*}}\right)+\left(N-N^{*} \ln \frac{N}{N^{*}}\right) .
\end{aligned}
$$

The dynamics of the ecoepidemic model is richer than the one of the purely demographic model. In the latter, there are transcritical bifurcations between the equilibrium with only bottom prey and the subsystem of the two bottom trophic layers, and between the latter and coexistence. In the ecoepidemic model, in addition to these, from the healthy-prey-only equilibrium the endemic bottom-prey-coexistence can emanate, if the prey carrying capacity is large enough. In (5.12) there are also persistent limit cycles, which are shown to be absent in the corresponding purely demographic case. Bistability is discovered among some equilibria, leading to situations in which the computation of their basins of attraction is relevant for the system outcome in terms of its biological implications, [28]. 


\subsection{Metaecoepidemic models}

Metapopulation, [62,63], is a tool introduced to study the influence of fragmented landscapes on the dynamics of wild populations, whether be it induced by human activies or natural causes. This concerns environmentalists and conservationists since landscape reshaping leading to heterogeneous environments may threaten population survival, [144].

Models introducing a disease in this context have been proposed in [137], with two possible habitats hosting a predator-prey ecoepidemic system, in which migrations are allowed only for the healthy prey. For instance, introducing the diseased prey in one patch and the predators in the other one, both unable to migrate, we are led to:

$$
\begin{aligned}
& N_{1}^{\prime}=r_{1} N_{1}\left(1-\frac{N_{1}}{K_{1}}\right)-a \frac{N_{1} Z}{H+N_{1}}-m_{21} N_{1}+m_{12} N_{2}, \quad Z^{\prime}=Z\left(\frac{e N_{1}}{H+N_{1}}-b\right), \\
& N_{2}^{\prime}=r_{2} N_{2}\left(1-\frac{N_{2}}{K_{2}}\right)-\gamma N_{2} I+\nu I+m_{21} N_{1}-m_{12} N_{2}, \quad I^{\prime}=I\left[\gamma N_{2}-\mu-\nu\right],
\end{aligned}
$$

the indices denoting the respective patches in which the populations thrive. In this model note the presence of the Holling type II response function to model predator's hunting. Note also that migrations from patch $i$ into patch $j \neq i$ are denoted by $m_{j i}, i, j=1,2$. In this case, the results show that patch 1 under suitable conditions allows persistent oscillations, when isolated. When communications between patches are allowed, these limit cycles could be either transferred to the second patch or not. Also disease eradication in the latter is possible, even though it is endemic when migrations are impossible. But it may also happen that in the coupled environment the oscillations are damped and the system settles to a stable coexistence equilibrium in both communicating environments. The predator-free equilibrium can also be attained, and the coupled system may also show the same behavior of each individual patch. These various outcomes are shown to arise for some realistic demographic parameter values related to two specific ecosystems. The first one is composed of Ovis canadensis, affected by nematode lungworms, Protostrongylus stilesi, together with its various predators such as wolf (Canis lupus), coyote (Canis latrans), bear (Ursus). In the second one the population of Strix occidentalis, with helmints as main parasites, is mainly predated upon by the great horned owl, Bubo virginianus. Purely hypothetical values were considered for the parameters that were not available.

An alternative situation considers instead the case of the disease present only in the patch were also predators are found, and the second patch being only a refuge for the susceptible prey:

$$
\begin{aligned}
& N_{1}^{\prime}=r_{1} N_{1}\left(1-\frac{N_{1}}{K_{1}}\right)-a \frac{N_{1} Z}{H+N_{1}}-m_{21} N_{1}+m_{12} N_{2}-\gamma N_{1} I+\nu I, \quad I^{\prime}=I\left[\gamma N_{1}-\mu-\nu\right], \\
& Z^{\prime}=Z\left(\frac{e N_{1}}{H+N_{1}}-b\right), \quad N_{2}^{\prime}=r_{2} N_{2}\left(1-\frac{N_{2}}{K_{2}}\right)+m_{21} N_{1}-m_{12} N_{2} .
\end{aligned}
$$

The findings in this case indicate that whenever the conditions for the predator-free equilibrium in patch 1 hold, the introduction of the healthy prey refuge causes an increase in the disease prevalence. Thus if the goal is fighting the disease, a safety refuge does not help and is to be avoided; rather, in the fragmented environment, the safety refuge should be eliminated to decrease the disease grip. But large differences in migration rates apparently influence this result, leading, from a predator-free equilibrium in patch 1 , to a disease-free equilibrium in the coupled model.

In general in the models considered in [137] coexistence of all the populations may not always be supported, depending on the system formulation.

A corresponding case has been considered in [21], where the disease affects the predators. Here, the prey are confined in patch 1 , the diseased predators in patch 2 , and the healthy predators can migrate 
between the two environments. The interactions are here expressed via Holling type I terms:

$$
\begin{aligned}
& N^{\prime}=r N\left(1-\frac{N}{K}\right)-a N P_{1}, \quad P_{1}^{\prime}=b N P_{1}-m P_{1}-n_{21} P_{1}+n_{12} P_{2}, \\
& P_{2}^{\prime}=-\gamma P_{2} Y-m P_{2}+n_{21} P_{1}-n_{12} P_{2}, \quad Y^{\prime}=\gamma P_{2} Y-\mu Y .
\end{aligned}
$$

Modifications of the environment may lead to unforseen consequences here as well, for which habitat fragmentation should be carefully analyzed before being implemented. In some cases however it could be a means of eradicating pests. Threshold parameters are identified, namely

$$
\mathcal{R}_{p} \equiv \frac{b K-n_{12}-n_{21}+\sqrt{\left(b K-n_{12}-n_{21}\right)^{2}+4 b n_{12}}}{2 m}, \quad \mathcal{R}_{d} \equiv \frac{r \gamma n_{21}\left[b K\left(m+n_{12}\right)-m\left(m+n_{12}+n_{21}\right)\right]}{\mu a K b\left(m+n_{12}\right)^{2}},
$$

and $\mathcal{R}_{0}^{m}=\min \left\{\mathcal{R}_{p}, \mathcal{R}_{d}\right\}$, the first one representing the value above which predators invade the metaecoepidemic system, $\mathcal{R}_{d}$ has the same role of $\mathcal{R}_{0}$ in standard epidemic models, while $\mathcal{R}_{0}^{m}>1$ is the condition for the disease to become endemic in this metaecoepidemic environment.

These specialist predators clearly would disappear in the absence of resources, but here migrations allow them to thrive also in the prey-free patch. Thus interrupting the communications between the two environments may lead to predators exitinction in harsh conditions, but it could be an option if diseaseeradication is sought, assuming however that the epidemic occurs only in the prey-free environment.

\subsection{Safety refuges}

We mentioned safety refuges in the previous subsection. Their role has also been recently investigated in the context of ecoepidemiology under a different framework. Some ideas dealing with this concept are here briefly discussed.

In [111] the functional form for the use of safety harbors is derived looking at the individual behavior, assuming that predators foraging occurs on a much shorter timescale that their lifespan, for which predators and prey can be considered constant populations at first, in these considerations. They are either hunting or handling a captured prey. The prey instead are either vulnerable or safe from attacks. A balance equation for the hunting predators is obtained, the rate of change in their numbers accounting for those that capture a prey, and therefore leave this class to handle it, and those that instead enter into it, after finishing the prey consumption. A similar equation is obtained for the prey that become vulnerable: they are incremented by those leaving the refuge and diminished by the ones that are captured by the predators' hunting. From these considerations, assessing the steady states, the functional response is determined resulting in a Beddington-De Angelis form, [16,46]. Two models are then considered, when the invulnerable prey do not reproduce, or, alternatively, when they do but their mortality rate is higher.

In [55] models are proposed for constant proportion of prey taking refuge:

$$
N^{\prime}=r N\left(1-\frac{N}{K}\right)-q Z \frac{N(1-\beta)}{N(1-\beta)+a}, \quad Z^{\prime}=b Z\left(p \frac{N(1-\beta)}{N(1-\beta)+a}-c\right)
$$

and for a constant number of prey finding harbor:

$$
N^{\prime}=r N\left(1-\frac{N}{K}\right)-q Z \frac{N-\gamma}{N-\gamma+a}, \quad Z^{\prime}=b Z\left(p \frac{N-\gamma}{N-\gamma+a}-c\right)
$$

Interestingly, the coexistence equilibrium is independent of the birth rates of both populations. The prey population at equilibrium increases, predators decrease, with a more frequent use of the refuges. The latter combined with low predators hunting efficiency leads to the predators disappearance. These models and some of their findings on the stabilizing effect of the refuges on the equilibrium have been questioned in [94] and further discussed in [56]. Note that a spatial version of the model (5.16) has been proposed and analyzed in [143] to show pattern formations in two dimensions. 
In the context of ecoepidemiology, [24] presents an extension of (3.1), [130], that includes a safety niche for the susceptibles:

$$
\begin{gathered}
S^{\prime}=S[a-b S-c Z]+\omega I-\lambda \max \{0,(S-s)\} I, \quad I^{\prime}=\lambda \max \{0,(S-s)\} I-I[k Z+\omega]-\mu I, \\
Z^{\prime}=Z[d+e S-f Z-h I]
\end{gathered}
$$

and then also the following corresponding cases are analyzed: for the refuge for the infected,

$$
\begin{gathered}
S^{\prime}=S[a-b S-c Z-\lambda \max \{0,(I-p)\}]+\omega I, \quad I^{\prime}=\lambda \max \{0,(I-p)\} S-I[k Z+\omega]-\mu I, \\
Z^{\prime}=Z[d+e S-f Z-h I],
\end{gathered}
$$

for reduced contacts

$$
\begin{gathered}
S^{\prime}=S[a-b S-c Z-(1-q) \lambda I]+\omega I, \quad I^{\prime}=I[(1-q) \lambda S-k Z-\omega-\mu], \\
Z^{\prime}=Z[d+e S-f Z-h I]
\end{gathered}
$$

and then also culling of the infected

$$
S^{\prime}=S[a-b S-c Z-\lambda I]+\omega I, \quad I^{\prime}=I[\lambda S-k Z-\omega]-(\delta+\mu) I, \quad Z^{\prime}=Z[d+e S-f Z-h I],
$$

Finally, a thorough analysis of the ecosystems' behavior in terms of the system parameters is performed.

\subsection{Chaotic behavior}

In epidemiology, more realistic models for the diseases that can be overcome and allow possible relapses are the so-called SIRS models. They contain also the class of recovered individuals $R$. These are the identified disease-carriers, that are therefore isolated so that they cannot transmit further the disease. Eventually, upon recovery, they would rejoin the susceptible class, if the disease has not given them immunity. In the realm of ecoepidemiology, the following very general ecoepidemic SIRS model with disease in the predators has been proposed, [125]:

$$
\begin{aligned}
& N^{\prime}=S N-G(N)(P+R+\alpha Y)-M_{N} N^{2}, \quad Y^{\prime}=\lambda(P, Y)-\left(M_{Y}+\mu\right) Y-\gamma Y, \\
& P^{\prime}=E G(N)(P+R+\alpha \beta Y)-M_{Y} P+\delta R-\lambda(P, Y), \quad R^{\prime}=\gamma Y-\delta R-M_{Y} R .
\end{aligned}
$$

The epidemics leaves the prey unaffected. $G$ and $\lambda$ are the functions representing predators hunting and disease transmission. The model is "normalized" around the coexistence equilibrium, denoted by stars in what follows, in the following way. Introduce $n:=N / N^{*}, p:=P / P^{*}, y:=Y / Y^{*}$ and $r:=R / R^{*}$ and define the normalized functional response $g(n):=G\left(N^{*} n\right) / G\left(N^{*}\right)$ together with the incidence $l(p, y):=$ $\lambda\left(P^{*} p, Y^{*} y\right) / \lambda\left(P^{*}, Y^{*}\right)$ functions, to obtain:

$$
\begin{aligned}
& n^{\prime}=a_{n}\left(n-\tilde{m}_{n} g(n)\left(\tilde{f}_{\alpha}(b p+\tilde{b} r)+f_{\alpha} y\right)-m_{n} n^{2}\right), \quad y^{\prime}=a_{i}(l(p, y)-y) . \\
& p^{\prime}=a_{s}\left(e_{s} g(n)\left(\tilde{f}_{\beta}(b p+\tilde{b} r)+f_{\beta} y\right)-m_{y} p+\tilde{e}_{s} r-\tilde{m}_{y} l(p, y)\right), \quad r^{\prime}=a_{r}(y-r) .
\end{aligned}
$$

The Jacobian of the normalized model is obtained in terms of the scale parameters and of the derivatives of the normalized processes at the steady state, that are defined as generalized parameters:

$$
g_{n}=\left.\frac{\partial g(n)}{\partial n}\right|_{n^{*}}, \quad l_{s}=\left.\frac{\partial l(p, y)}{\partial p}\right|_{p^{*}, y^{*}}, \quad l_{i}=\left.\frac{\partial l(p, y)}{\partial y}\right|_{p^{*}, y^{*}} .
$$

Computing the stability of the coexistence equilibrium, two surfaces are found, both arising with simpler shape also in the disease-free model, containing respectively saddle-node type and Hopf bifurcations. The latter has the shape of a Whitney umbrella. Along the line in which the Hopf bifurcation surface intersects itself, there are two pairs of purely imaginary eigenvalues. At the intersections with the saddlenode bifurcation surface, there are the lines of a Takens-Bogdanov bifurcation, existing already in the 
purely demographic model, and of a Gavrilov-Guckenheimer bifurcation. The latter arises from a triple point bifurcation on the Takens-Bogdanov bifurcation line; along it, the Jacobian has a zero and two purely imaginary eigenvalues, indicating that quasiperiodic and chaotic dynamics are likely to occur, while chaotic behavior is expected from the presence of the double Hopf bifurcation.

The model is specified using $g(n)=a n^{2}\left(1+b n^{2}\right)^{-1}$ and $l(p, y)=c p y(1+d p+e y)^{-1}$ as response and incidence functions. Technical conditions arise from normalization, i.e. $g(1)=1$ and $l(1,1)=1$, leading respectively to $a=(1+b)$ and $c=(1+d+e)$, so that $g_{n}=2(1+b)^{-1}, l_{s}=1+e(1+d+e)^{-1}$ and $l_{i}=1+d(1+d+e)^{-1}$. Then, Neimark-Sacker bifurcations are discovered, where a limit cycle becomes unstable giving rise to a stable quasiperiodic motion on a torus, leading ultimately to a route to chaos. The parameter ranges for which these results hold are rather large, so that these phenomena should not be easily neglected. Note that this behavior cannot occur neither in the purely demographic model, nor in the purely SIRS epidemic system, while chaotic behavior is a generic property of this type of models. But the presence of the recovered class is essential, because this behavior has not been found in the ecoepidemic systems just of type SIS.

Observe, however, that the most frequently observed route to chaos is, however, via period-doubling, which has been observed in $[12,14,119,128]$.

\section{Effects of time lags}

There are different ways in which a system may show retarded responses, thus we consider here delayed models in strict sense, but also models in which there is a class of "exposed" individuals to the disease, that are not yet infectious.

For instance the delay can be due to the so-called "handling time" in processing the prey that a predator has captured, essentially to digest it. This in general is taken care of by the HTII term, the half saturation constant indeed accounting for this time. For diseases, in general, an individual shows the symptoms after some time from becoming infected, but, what is more important, becomes infectious not immediately after disease contraction. This incubation period can be modeled by introducing a delay $\tau$ for the new recruitments in the infected class at time $t$, [74]. They enter this class if they were subject to contagion at time $t-\tau$. Finally, in classical epidemiological models, there is the alternative of introducing a class of exposed individuals, in which the disease is incubating, for which they are not yet infectious. But this class if often omitted in epidemic models since the latency is not crucial for the disease propagation, [73].

In [66] the following delayed ecoepidemic model with disease in the predators is considered, taking into account the time needed for the predators to digest their prey and generate newborns, $\mu \in \mathbf{R}$ expressing the predators net birth rate:

$$
\begin{aligned}
& N^{\prime}=r N\left(1-\frac{N}{r / B}\right)-m N P-p m N Y=r N-B N^{2}-m N P-p m N Y, \\
& P^{\prime}=e m N(t-\tau) P(t-\tau)-a P Y+\mu P(t-\tau), \quad Y^{\prime}=a Y P+e m p N(t-\tau) Y(t-\tau)-\delta Y .
\end{aligned}
$$

The initial conditions $\phi=\left(\phi_{1}, \phi_{2}, \phi_{3}\right)$ are defined in $C_{+}=\left\{\phi \in C\left([-\tau, 0], \mathbf{R}_{+}^{3}\right): \phi_{1}(\theta)=N(\theta), \phi_{2}(\theta)=\right.$ $\left.P(\theta), \phi_{3}(\theta)=Y(\theta)\right\}$, where $N(\theta)>0, P(\theta)>0, Y(\theta)>0, \theta \in C[-\tau, 0]$ are given functions. The system's equilibria both in the absence and in the presence of the delay are analyzed.

In case of no delay the disease-free equilibrium is feasible only for $\mu<0$ if $\mathrm{erm}>-b \mu$, entailing for the predators either a high enough total mortality rate or a low hunting rate; it is conditionally stable, but no Hopf bifurcations can arise. When the delay is introduced, there is instead a critical value $\tau^{0}$ for the delay, for which a supercritical Hopf bifurcation occurs when $\tau$ grows past it. A similar result, with a different threshold $\tau^{*}$, holds also for the coexistence equilibrium with endemic disease. The difference is that in the model with no delay this equilibrium is found in both cases $\mu>0$ and $\mu<0$, with different feasibility conditions, but it is unconditionally locally asymptotically stable. Conditions on the permanence of the system are derived both in the absence and in the presence of the delay. 
A different idea is explored in [135], where the prey exposed class $E$ is introduced. The basic model is:

$$
\begin{aligned}
& S^{\prime}=(r-n) S+r(1-\delta) E+r(1-\theta) I-\gamma S I-a S Z, \quad E^{\prime}=\gamma S I-(n+\alpha) E-b E Z, \\
& I^{\prime}=\alpha E-(n+\mu) I-c I Z, \quad Z^{\prime}=e(a S+b E+c I) Z-m Z .
\end{aligned}
$$

Center oscillations are found, inherited from the underlying Lotka-Volterra demographic structure of the model. The predator-free equilibrium is unconditionally unstable, which corresponds to the endemic equilibrium of an SEI model. Introduction of predators in such a situation completely changes the ecosystem dynamics. In the same paper, [135], several other variations with intraspecific competition are considered, starting from:

$$
\begin{aligned}
& S^{\prime}=(r-n) S\left(1-\frac{\eta S+\xi E+\omega I}{K}\right)-\gamma S I-a S Z, \\
& E^{\prime}=\gamma S I-(n+\alpha) E-\pi(r-n) \frac{\eta S+\xi E+\omega I}{K} E-b E Z, \\
& I^{\prime}=\alpha E-(n+\mu) I-\sigma(r-n) \frac{\eta S+\xi E+\omega I}{K} I-c I Z, \quad Z^{\prime}=[e(a S+b E+c I)-m] Z .
\end{aligned}
$$

In this very general form, the suitable tuning of the parameters $\eta, \xi, \omega, \sigma, \pi$ allows to consider various cases for selective hunting, expressing the facts that predators may avoid the diseased less palatable prey, or instead capture them because they are weaker. Persistent oscillations are discovered and appear to be related to the larger number of subpopulations present in this kind of system.

The corresponding model with exposed class $F$ among the predators is presented in [126]:

$$
\begin{aligned}
& N^{\prime}=r N\left(1-N K^{-1}\right)-a S N-b F N-c I N, \\
& S^{\prime}=-m S+a e N S+b e(1-\delta) F N+c e(1-\theta) I N-\gamma S I+\rho I, \\
& F^{\prime}=\gamma S I-(m+\alpha) F, \quad I^{\prime}=\alpha F-\mu I-m I-\rho I .
\end{aligned}
$$

In it, the predator's disease-free reproduction number $R_{0}^{p}=a e \mathrm{Km}^{-1}$ and the epizootiological reproduction numbers for exposed and infected, respectively $\mathcal{R}_{0}^{F}=\gamma(m+\alpha)^{-1}$ and $\mathcal{R}_{0}^{I}=\alpha(m+\mu+\rho)^{-1}$, are derived. Letting $\mathcal{R}_{0}=\mathcal{R}_{0}^{F} \mathcal{R}_{0}^{I}$, The stability of the disease-free predator-prey equilibrium holds for $\mathrm{raR}_{0}{ }^{-1}\left(1-\left(R_{0}^{p}\right)^{-1}\right)<1$. It is thus enhanced by a suitably high prey reproduction rate or by a low healthy predators hunting rate. In the corresponding model without incubation class, the epizootiological reproduction number is instead $\mathcal{R}_{0}^{*}=\gamma(m+\mu+\rho)^{-1}$. Again, the stability condition of the disease-free equilibrium becomes $\mathrm{ra}\left(\mathcal{R}_{0}^{*}\right)^{-1}\left(1-\left(R_{0}^{p}\right)^{-1}\right)<1$ and once more a highly reproducing prey allows the epidemics in the predators to become endemic. The limit cycles that this model exhibits are found also in a simpler model, thereby suggesting that this kind of dynamics may depend more on the higher dimensionality of the system rather than to the presence of the latency class.

In [10] a model with mass action incidence and HTII hunting on both healthy and infected prey is presented, in which a distributed delay on the infection process is considered:

$$
\begin{aligned}
& S^{\prime}=S\left[\left(1-\frac{S+I}{K}\right)-\lambda I-n \frac{Z}{a_{1}+S}\right], \quad I^{\prime}=\lambda \int_{-\infty}^{t} S(u) I(u) f(t-u) d u-I\left[\frac{m Z}{a+I}+\mu\right], \\
& Z^{\prime}=Z\left[\frac{m \alpha I}{a+I}+\frac{n \alpha S}{a_{1}+S}-d\right], \quad f(u)=\frac{a^{p+1} u^{p}}{p !}
\end{aligned}
$$

and $f$ represents the waning in time of infectivity of the infectious individuals. The average delay is $\bar{T}=\int_{0}^{\infty} u f(u) d u=(p+1) a^{-1}$. Using the delta function $f(u) \delta(u-\tau)$ for the kernel, system (6.5) gets transformed into the following delay differential system:

$$
\begin{aligned}
& S^{\prime}=S\left[\left(1-\frac{S+I}{K}\right)-\lambda I-n \frac{Z}{a_{1}+S}\right], \quad I^{\prime}=\lambda I(t-\tau) S(t-\tau)-I\left[\frac{m Z}{a+I}+\mu\right], \\
& Z^{\prime}=Z\left[\frac{m \alpha I}{a+I}+\frac{n \alpha S}{a_{1}+S}-d\right], \quad S(\theta)=\varphi(\theta), \quad I(\theta)=\psi(\theta), \quad S(\theta)=\xi(\theta), \quad \theta \in[-\infty, 0] .
\end{aligned}
$$


The system is analysed when predators capture only infected prey, i.e. for $n=0$. Coexistence is stable in the no-delay system if the basic reproductive ratio $R_{0}=\lambda K \mu^{-1}$ exceeds 1 and the hunting rate is within a certain interval. For the system with delay, there is another technical condition to be satisfied, and then stability or instability follows from the size of the delay being lower or respectively exceeding a certain threshold. For the delay coinciding with the threshold, a Hopf bifurcation occurs, which may lead to large oscillations that are prone to produce population extinction under possible system perturbations. Numerical experiments indicate some criteria for acting on the model parameters in order to dampen or eliminate these oscillations. Among these, there could be a larger growth rate of the predator population. It can be achieved e.g. by allowing generalist predation, i.e. hunting not only infected but also healthy prey. Alternatively, providing an additional food source could lead to similar results. Note finally that if the infection rate is not large, the system stabilizes at coexistence independently of the delay, but for larger values, the threshold phenomenon occurs.

In [30] a time-delay extension of the model presented in [29] is considered, accounting for the digestion time $\tau, t_{\tau}=t-\tau$, of the infected prey, that have a fatal effect on the predators. The nondimensional model is:

$$
S^{\prime}=B-S[\sigma I-\alpha Z-\delta]+\mu I, \quad I^{\prime}=I[\sigma S-\beta Z-\gamma-\mu], \quad Z^{\prime}=Z[1-Z+\alpha S]-\beta Z\left(t_{\tau}\right) I\left(t_{\tau}\right)
$$

and suitable initial conditions $S(t)>0, I(t)>0, Z(t)>0$, for $t \in[-\tau, 0]$. The delay plays no role both in the healthy-prey-only and the disease-free equilibria. Conditions are derived ensuring that the coexistence equilibrium remains asymptotically stable in an interval for the delay parameter, providing an explicit estimate for its maximum value, $\tau_{+}$. In suitable conditions the coexistence equilibrium can be shown to be stable for every $\tau>0$; if for a certain $\tau_{0}$ it is unstable, it is however unstable from there onwards, i.e. for all $\tau>\tau_{0}$. Also, Hopf bifurcations leading to small amplitude periodic solutions exist depending on the size of the delay. Thus the digestion time may help in the disease control. Global stability for the disease-free state is proven via a suitable Lyapunov function as well as system permanence, but here the time lag does not play any role.

Finally, in [83], the following model is presented:

$$
S^{\prime}=r S\left[1-\frac{S}{K}\right]-\lambda \frac{S I(t-\tau)}{a+S}-c \frac{S Z}{b+S}, \quad I^{\prime}=\lambda \frac{S I(t-\tau)}{a+S}-\mu I, \quad Z^{\prime}=Z\left[e c \frac{S}{b+S}-d Z-m\right] .
$$

It accounts for logistic growth of the prey in which only healthy individuals reproduce, delayed disease transmission with a frequency dependent-like transmission, Holling type II predation; $\mu$ represents natural plus disease-related mortality for the infected prey; the predators suffer from intraspecific competition in addition to natural mortality. Boundedness of the trajectories, stability of the coexistence equilibrium and Hopf bifurcations are investigated. In [93] this model is extended with a second delay to account for the time needed for the assimilation of the captured prey. However, with respect to the latter, the time lag in the disease transmission appears to have a predominant role for the systems' dynamics.

\section{Aquatic ecoepidemic models}

The model (4.12) could be considered as a transition toward the class of models in the aquatic realm.

The first model in such context is [17], which is also a model for standard disease incidence. It contains healthy $S$ as well as virally infected $I$ phytoplankton and zooplankton $Z$. Logistic growth of the healthy phytoplankton is assumed, grazing occurs via a mass action law, the disease as said is transmitted via standard incidence:

$$
S^{\prime}=r S\left[1-\frac{S+I}{K}\right]-a S Z-b \frac{S I}{S+I}, \quad I^{\prime}=b \frac{S I}{S+I}-q I Z-c I, \quad Z^{\prime}=a S Z+q I Z-d Z .
$$

The paper discusses occurrences of oscillations, possibly chaotic, for this system and compares actual data for recurring outbreaks of Noctiluca scintillans and diatoms. 
In [35] harvesting, with $E_{i}, i \in\{S, Z, I\}$ being the harvest rates, is introduced into the picture leading to the following model:

$$
\begin{aligned}
& S^{\prime}=\alpha S\left(1-\frac{S}{K}\right)-\frac{\beta S Z}{1+a S}-E_{S} S-\lambda S I, \quad I^{\prime}=I\left(\lambda S-\delta Z-\mu-E_{I}\right), \\
& Z^{\prime}=\gamma Z+\frac{c \beta S Z}{1+a S}-E_{Z} Z+\delta Z I .
\end{aligned}
$$

There is a transcritical bifurcation between the healthy-prey only equilibrium and the disease-free equilibrium. Persistence is shown if the contact rate $\lambda$ lies in a suitable interval. Within this range, there exists a critical threshold $\xi_{0}$ for the healthy prey population size at the coexistence equilibrium so that a Hopf bifurcation occurs whenever this population becomes larger than $\xi_{0}$. Numerical evidence indicates that the persistent oscillations can be damped with the system settling to coexistence whenever the removal of infected prey $E_{I}$ is large enough.

After rescaling, the model of [110] is:

$$
\begin{aligned}
& S^{\prime}=S(1-S-I)-\theta_{1} S I-\theta_{2} Z S, \quad I^{\prime}=\theta_{1} S I-\theta_{3} I-\eta_{1} Z I, \\
& Z^{\prime}=\theta_{4} Z(1-Z)-\theta_{5} Z+\theta_{6} Z S+\eta_{2} Z I .
\end{aligned}
$$

Here, predators have an alternative food supply and select to hunt only healthy prey, initially $\eta_{1}=\eta_{2}=$ $\theta_{5}=0$. Criteria for the extinction of susceptible and infected prey are provided, involving these two subpopulation initial values. Disease-selective hunting entails a high risk of prey extinction and does not allow coexistence of all these populations. Global asymptotic stability of the coexistence equilibrium is shown for the full model using a suitable Lyapunov function.

In [37] also the role of nutrients, basic compounds $C$ that are assimilated by the phytoplankton, is explicitly taken into account, while the phytoplankton is subject to a transmissible disease. The first model is:

$$
\begin{aligned}
& C^{\prime}=\left(C^{0}-C\right) D-a S u(C)-b I v(C), \quad S^{\prime}=a S u(C)-\frac{\lambda S I}{S+I}-D_{1} S, \\
& I^{\prime}=b I v(C)+\frac{\lambda S I}{S+I}-D_{2} I,
\end{aligned}
$$

$C^{0}$ representing the nutrients supply into the environment. The nutrient uptake rates $u$ and $v$ are continuous functions through the origin, steadily increasing with horizontal asymptote at 1 . Criteria for the extinction of both susceptible and infected phytoplanktons are derived, respectively being $R_{0}=$ $a u\left(C^{0}\right) D_{1}^{-1}<1$ and $\widetilde{R}_{0}=b v\left(C^{0}\right)\left(D_{2}-\lambda\right)^{-1}<1$. The disease-free and the stability of the pandemic (i.e. healthy phytoplankton-free) equilibria is characterized. The second model specializes the choice of $u$ and $v$ to Holling type II response functions:

$$
\begin{aligned}
& C^{\prime}=\left(C^{0}-C\right) D-\frac{a S C}{K_{1}+C}-\frac{b I C}{K_{2}+C}, \quad S^{\prime}=\frac{a S C}{K_{1}+C}-\frac{\lambda S I}{S+I}-D_{1} S, \\
& I^{\prime}=\frac{b I C}{K_{2}+C}+\frac{\lambda S I}{S+I}-D_{2} I .
\end{aligned}
$$

The plankton-free equilibrium is stable whenever $R_{0}=a C^{0}\left[D_{1}\left(K_{1}+C^{0}\right)\right]^{-1}<1$ and $\widetilde{R}_{0}=b C^{0}\left[\left(D_{2}-\right.\right.$ $\left.\lambda)\left(K_{1}+C^{0}\right)\right]^{-1}<1<D_{2}\left(D_{2}-\lambda\right)^{-1}$. For $R_{0}>1$ and $\widetilde{R}_{0}>D_{2}\left(D_{2}-\lambda\right)^{-1}$ there are the diseasefree equilibrium and a healthy phytoplankton-free state. The former is stable if $R_{0}>1, a D_{1}^{-1}>1$ and $R_{1}=b C_{1}\left[\left(D_{2}-\lambda\right)\left(K_{2}+C^{1}\right)\right]^{-1}<1$, while the latter is for $\widetilde{R}_{0}>D_{2}\left(D_{2}-\lambda\right)^{-1}, b D_{2}^{-1}>1$ and $\widetilde{R}_{1}=a C_{2}\left[\left(D_{1}+\lambda\right)\left(K_{1}+C^{2}\right)\right]^{-1}<1$, where $C_{1}$ and $C_{2}$ are the levels of nutrients at these points. For $R_{1}>1$ or $\widetilde{R}_{1}>1$ coexistence occurs but it is unstable. Finally, the model in which the infected cannot feed is presented, setting $b=0$ in (7.5). In this case the coexistence steady state is instead a global attractor. 
In [124] phytoplankton is affected by a transmissible disease and grazed upon by the zooplankton:

$$
S^{\prime}=a S\left[a-b \frac{S+I}{a}\right]-c S Z-\lambda S I, \quad I^{\prime}=I(\lambda S-k Z-h), \quad Z^{\prime}=Z\left(-d+e S+k_{1} I\right) .
$$

Viruses are not explicitly taken into account. Local stability analysis is performed showing some transcritical bifurcations among the equilibria. Hopf bifurcations are analytically shown to occur and their peaks are qualitatively compared to actual blooms data. Interestingly, for (7.6) chaotic behavior is discovered in [128].

The paper [123], see also [96], is among the first models with highly nonlinear incidence and response functions, for which for an explicit carrying capacity there is no coexistence of healthy and infected prey together with predators. In nondimensional form, the model is:

$$
\begin{aligned}
& N^{\prime}=N\left[(1-N)\left[1-(1-\rho) i-\mu_{I} i\right]-\frac{\alpha^{m} N^{m} Z}{1+\beta^{m} N^{m}}, \quad i^{\prime}=i(1-i) r_{i}\left(Z-Z_{2}\right),\right. \\
& Z^{\prime}=\frac{\mu_{Z} Z\left(Z^{m}-Z_{1}^{m}\right)}{Z_{1}^{m}\left(1+\beta^{m} N^{m}\right)},
\end{aligned}
$$

where $m=1$ gives the HTII response function, $m=2$ the HTIII one and $r_{i}=1-\rho+\lambda$ holds for mass action incidence, while $r_{i}=1-\rho$ for standard incidence. Also, the system's steady states change depending on the type of disease transmission used, namely $Z_{2}=\left(\mu_{I}+1-\rho\right)(\lambda+1-\rho)^{-1}$ for mass action, $Z_{2}=1-\left(\lambda-\mu_{I}\right)(1-\rho)^{-1}$ for standard incidence, $Z_{1}=\mu_{Z}\left(\alpha-\beta \mu_{Z}\right)^{-1}$ for HTII and $Z_{1}=$ $\left[\mu_{Z}\left(\alpha^{2}-\beta^{2} \mu_{Z}\right)^{-1}\right]^{\frac{1}{2}}$ for HTIII. The possible stable ecosystem outcomes are described in diagrams as functions of the disease-free and predator-free subsystems. The models are then generalized to explicitly account for viral particles, as in [122], including also the HTIII functional response.

But in contrast to [123], in [101] coexistence of $S, I$ and $Z$ becomes instead possible when the disease transmission depends on the predator. Consider

$$
\begin{aligned}
& S^{\prime}=S\left(1-\frac{S+I}{K}\right)-\lambda(Z) S I-b S Z, \quad I^{\prime}=\lambda(Z) I S-D I-a I Z \\
& Z^{\prime}=\left(\omega_{S} f_{S}(S, I)+\omega_{I} f_{I}(S, I)-\delta\right) Z
\end{aligned}
$$

with the disease transmission rate being linearized, $\lambda(Z)=\lambda_{0}+\alpha Z$, where $\alpha \in R$ represents the predators' influence. The cases of predators feeding only on infected or healthy prey, respectively $b=0$ and $a=0$, are at first examined and then the combined case. The emergence of a disease-induced strong Allee effect for the predators is shown. Among the other many interesting findings, the predators influencing the disease transmission can enhance the predators survival, if they do not feed on other resources; however, it can also favor the spreading of the epidemics, that otherwise would be eradicated and increase infection prevalence; for systems with large prey carrying capacity, i.e. eutrophication, it may further have a destabilizing effect, potentially leading to the ecosystem extinction; finally, in these conditions, predators can survive if their initial state lies within the domain of attraction of a suitable equilibrium, in view of a bistability phenomenon. In this respect, we mention that the accurate calculation of separatrix surfaces has been dealt with in [28].

For the following phytoplankton-zooplankton model with infection in the former, [78], with HTII grazing and standard incidence:

$$
\begin{aligned}
& S^{\prime}=r_{1} S(1-S-I)-a \frac{S Z}{1+b(S+I)}-\lambda \frac{S I}{S+I}, \\
& I^{\prime}=r_{2} I(1-S-I)-a \frac{I Z}{1+b(S+I)}+\lambda \frac{S I}{S+I}-m_{2} I, \quad Z^{\prime}=a \frac{(S+I) Z}{1+b(S+I)}-m_{3} Z,
\end{aligned}
$$


stationary and nonstationary oscillations are discovered in the transformed model using the total phytoplankton population $N=S+I$ and the prevalence $i=I N^{-1}$ as new variables:

$$
\begin{aligned}
& N^{\prime}=\left[r_{1}(1-i)+r_{2} i\right] N(1-N)-a \frac{N Z}{1+b N}-m_{2} i N, \\
& i^{\prime}=\left[\left(r_{2}-r_{1}\right)(1-N)+\left(\lambda-m_{2}\right)\right] i(1-i), \quad Z^{\prime}=a \frac{N Z}{1+b N}-m_{3} Z,
\end{aligned}
$$

Among the others, a continuum of equilibria is found, when the parameters satisfy $1-\left(\lambda-m_{2}\right)\left(r_{1}-r_{2}\right)^{-1}=$ $m_{3}\left(a-m_{3} b\right)^{-1}$, which lies on a heteroclinic line joining two saddle-foci semitrivial equilibria, namely the zooplankton-free and the disease-free points. There is a zip bifurcation, given by the singular curve folding into periodic solutions as a certain parameter changes. When the parameters do not satisfy the above relation, by increasing virulence, the trajectory approaches a "strange periodic attractor", which is not chaotic and allows coexistence of the three populations in a wide parameter range.

While (7.9) was used to model a lytic viral reproduction cycle, it is worth noting that other viral reproduction cycles have been considered as well. In [95] a model with lysogeny was studied, whereas in [79] a stochastic switch between lysogenic and lytic reproduction cycles has been modeled. In this context we cite also the classical Beretta-Kuang model [19] for viral infection in an aquatic environment: viruses $V$ and bacteria, $S$ and $I$, as their hosts are explicitly built into the model. In [122] also the confined viruses $W$ within the bacteria are accounted for:

$$
\begin{aligned}
& V^{\prime}=-\lambda S V-m_{V} V+\epsilon m_{I} W, \quad W^{\prime}=a W-c \frac{W^{2}}{I}-\epsilon m_{I} W+\lambda S V, \\
& S^{\prime}=r S\left(1-\frac{S+I}{K}\right)-\lambda S V, \quad I^{\prime}=\lambda S V-m_{I} I .
\end{aligned}
$$

Although not completely pertaining to our field of ecoepidemiology, in this context we mention also the recent interesting paper [102] on the emergence of a Holling type III response function in plankton dynamics. Finally, despite the fact that in this paper we do not consider spatial models, we briefly mention that in [39] zooplankton is assumed to feed on two phytoplankton populations. When one of them is toxic, it produces inhomogeneities in the spatial zooplankton distribution. Further, in [117] the travelling waves from which these patterns originate are shown, with their dependence on the diffusion coefficients.

Finally, the role of toxic algae in the occurrence of red, or brown, tides have been investigated in several papers. These do not strictly pertain to the class of ecoepidemic models, as the toxins do not infect non-toxic algae by contact to as diseases do, but they are related concepts and of interest here as well. Thus we will discuss one of them, and briefly mention some others without explicitly writing the equations.

Several two-population toxic producing phytoplankton $T$ - zooplankton $Z$ models are studied in [36], from the following basic system:

$$
T^{\prime}=r T\left(1-\frac{T}{K}\right)-\alpha f(T) Z, \quad Z^{\prime}=\beta f(T) Z-\mu Z-\theta g(T) Z,
$$

for various choices of the response functions $f$ and $g$, showing the existence of planktonic blooms and their termination due to the effect of the toxins release. In fact, the coexistence equilibria are analyzed for feasibility and the analytic conditions for the occurrence of Hopf bifurcations are derived in most cases. Thus the toxic producing phytoplankton not only controls the growth of other species, but exhibits also properties of self regulation.

The concept of "herd behavior", see Section 8.1, has also been exploited in the context of plankton modeling, [34], in this case using a different nonlinear function. Note that this functional response has already been employed in [107]. With the exponent $2 / 3$, the latter accounts for the spatial, i.e. threedimensional, nature of the situation. This is a consequence of the surface of the spherical patches of a 
phytoplankton population $\Pi$ being proportional to $\left[\frac{1}{N} k \Pi\right]^{\frac{2}{3}}=\rho \Pi^{\frac{2}{3}}, \rho \equiv\left(\frac{k}{N}\right)^{\frac{2}{3}}$. The model may lead to a possible explanation of harmful monospecies blooms.

Several models for harmful algal blooms are investigated in [107], which contains further references on earlier ecological plankton models. The systems presented account respectively for independent, competing and independently growing and competing phytoplankton populations. The models exhibit the same equilibria, with some changes for feasibility and stability. Limit cycles are found experimentally, with the unstable ones in the first model ultimately colliding with the stable ones thereby turning off the harmful blooms.

Two competing harmful phytoplankton populations and one zooplankton population are considered in [113]. The presence of the two toxin releasing phytoplankton populations reduces the equilibrium population values, helping the reduction of planktonic blooms. Alternatively, if one phytoplankton population is harmless, [109], toxin allelopathy is found to reduce the phytoplankton's interspecific competition and to flounder the growth of the zooplankton population.

The model in [11] considers zooplankton feeding again on healthy and toxic phytoplankton, but the main assumption is that the zooplankton upon ingestion recognizes the toxic phytoplankton and decreases its consumption. Mathematically, this is rendered via a Monod-Haldane type functional response, raising up to a maximum, and then decreasing again for larger values of the toxic phytoplankton population. The system dynamics is confined within a compact set near the origin. Nontoxic phytoplankton outcompetes the other ones if zooplankton grazes sufficiently on the toxic phytplankton, and its intrinsic reproduction rate coming from nontoxic phytoplankton ingestion is lower than its mortality rate. The toxic phytoplankton-only equilibrium can coexist with the former. It needs a low enough reproduction rate of nontoxic phytoplankton. To diminish the risk of brown tide outbreaks, the toxic phytoplankton carrying capacity must be kept small, by reducing nutrients in the environment. Transcritical bifurcations relate the first equilibrium with the toxic phytoplankton-free equilibrium. Note also that the equilibrium with no nontoxic phytoplankton is impossible, because the zooplankton would then disappear. The toxic phytoplankton can invade the nontoxic phytoplankton environment if it possesses a high reproduction rate. No system oscillations are possible near all these equilibria, but they are numerically found involving all the three populations, which alternatively can coexist also at a stable state. Thus refraining zooplankton from feeding on toxic phytoplankton does not prevent the insurgence of brown tides. The major discovery of the model is that the zooplankton does not disappear due to a large phytoplankton population, because the zooplankton's grazing pressure decreases with the increasing toxic species densities.

In [118] in the presence of toxins the grazing is instead reduced for both phytoplankton populations. The equilibria are the same as those of [11] but their analysis gives more stringent feasibility and stability conditions.

\section{Fairly recent issues}

\subsection{Herd behavior}

In [1] the interactions among predators and prey have been modeled using the rather different concept of herd behavior, some related ideas appearing in [42]. More elaborated demographic social models allow the individuals of the prey population to gather together to share the pasture, while the predators still use an individualistic behavior. Also symbiotic and competing demographic interactions are investigated. The model suitably accounts for the fact that interactions then occur mainly at the perimeter of the ground occupied by the prey. Interpreting the population $A$ as a density over the ground that it occupies, its portion lying on the perimeter must evidently be proportional to the square root of the density, $\sqrt{A}$, as that is the relationship between the two dimensional surface and the linear manifold describing its boundary. The system exhibits unexpected behaviors: for instance, competitive exclusion is prevented, because the socialized herd behavior preserves from extinction the competing individualistic population. For the predator-prey situation, limit cycles arise, which are shown to be nonexistent in the classical 
quadratic model. An application of this approach to the population invasion problem is contained in [20], for the case of the European hare replacing the native mountain hare in Italy.

These concepts are extended to the ecoepidemic case in [136]. Denoting by $\widehat{S}$ the healthy prey, by $\widehat{I}$ the infected prey and by $\widehat{Z}$ the predators' population, assuming that the diseased individuals are left behind and therefore are still subject to individual attacks, the system is:

$$
\frac{d \widehat{S}}{d \tau}=r \widehat{S}\left(1-\frac{\widehat{S}}{K}\right)-\widehat{\lambda} \widehat{S} \widehat{I}-\widehat{a} \sqrt{\widehat{S}} \widehat{Z}, \quad \frac{d \widehat{I}}{d \tau}=\widehat{I}(\widehat{\lambda} \widehat{S}-\widehat{b} \widehat{Z}-\widehat{\mu}), \quad \frac{d \widehat{Z}}{d \tau}=\widehat{Z}(\widehat{a} \widehat{e} \sqrt{\widehat{S}}+\widehat{b} \widehat{e} \widehat{I}-\widehat{m}) .
$$

There is a singularity in the Jacobian when the healthy prey population vanishes; therefore, to remove it, a change of dependent variables is performed introducing $\widehat{Q}=\sqrt{\widehat{S}}$. Further rescaling leads to the following nondimensional system for $Q>0$ :

$$
Q^{\prime}=Q\left(1-Q^{2}\right)-a Z-\lambda Q I, \quad I^{\prime}=I\left(\lambda Q^{2}-b Z-\mu\right), \quad Z^{\prime}=Z[e(a Q+b I)-m] .
$$

The analysis can be performed introducing the fundamental parameters $\rho=m(a e)^{-1}, \mathcal{R}_{p}=\rho-1$ and $\mathcal{R}_{0}=\lambda \mu^{-1}$. The healthy prey-only equilibrium exists always and is stable for $\mathcal{R}_{p}<1, \mathcal{R}_{0}<1$. The disease-free equilibrium is feasible for $\mathcal{R}_{p} \geq 1$, the predator-free one if $\mathcal{R}_{0} \geq 1$, showing transcritical bifurcations among the last two equilibria and the healthy prey-only one. The disease-free equilibrium is stable if $\mathcal{R}_{p}<\sqrt{3}$, a condition that arises already in [1], plus another one a bit more involved:

$$
R_{0}^{A}=\max \left\{\frac{m^{2}\left(a^{2} e \lambda+b m\right)}{a^{2} e^{2}\left(a^{2} e \mu+b m\right)}, \frac{a e}{\sqrt{3} m}\right\}=\max \left\{\rho^{2} \frac{(a \lambda+b \rho)}{(a \mu+b \rho)}, \frac{1}{\sqrt{3} \rho}\right\}<1 .
$$

The predator-free equilibrium is stable just for:

$$
R_{0}^{B}=\frac{\lambda^{2} m+b e \mu}{\lambda e(a \sqrt{\lambda \mu}+b)}<1 .
$$

Feasibility conditions for the unique coexistence equilibrium $\left(Q_{4}, I_{4}, Z_{4}\right)$ are:

$$
\rho^{2}\left(\frac{a \mu}{b \rho^{3}}-1\right)=\frac{m^{2}}{a^{2} e^{2}}\left(\frac{a^{4} e^{3} \mu}{b m^{3}}-1\right) \leq \frac{\lambda m}{b e}-1 \leq \mu\left(\frac{a}{b} \sqrt{\frac{\lambda}{\mu}}-\frac{1}{\lambda}\right),
$$

or more simply:

$$
\frac{m}{a e}=\rho \geq Q_{4} \geq \sqrt{\frac{\mu}{\lambda}} .
$$

Limit cycles are numerically shown to arise.

In [26] the model (8.1) is modified to take into account that infected individuals may remain in the herd. The formulation is:

$$
\begin{aligned}
& \frac{d S}{d t}=r S\left(1-\frac{S+I}{K}\right)-\sigma \frac{S I}{S+I}-q P \frac{S}{S+I} \sqrt{S+I}, \quad \frac{d I}{d t}=\sigma \frac{S I}{S+I}-w P \frac{I}{S+I} \sqrt{S+I}-\mu I, \\
& \frac{d P}{d t}=-m P+g P \frac{S}{S+I} \sqrt{S+I}+f P \frac{I}{S+I} \sqrt{S+I} .
\end{aligned}
$$

Note that the last term of the first equation expresses the capture of healthy prey on the edge of the herd by the predators, at rate $q$. In fact, in this term the last two expressions tell how many sound prey are on the border: the boundary population is $\sqrt{S+I}$ as we already know, of which only $S(S+I)^{-1}$ are healthy individuals. The corresponding dual fraction $I(S+I)^{-1}$ gives instead the infected individuals on the boundary in the predation term of the second equation. 
A series of substitutions leads to the following reformulation

$$
\begin{aligned}
& \frac{d A}{d t}=(\sigma-r-\mu) A^{2}+\frac{r}{K} A^{2} T^{2}+(q-w) A^{2} U+(r+\mu-\sigma) A-\frac{r}{K} A T^{2}+(w-q) A U, \\
& \frac{d T}{d t}=-\frac{r}{2 K} A T^{3}-\frac{\mu}{2} T+\frac{r+\mu}{2} A T-\frac{w}{2} U T+\frac{w-q}{2} A U T, \\
& \frac{d U}{d t}=\frac{w}{2} U^{2}+\frac{q-w}{2} A U^{2}+\left(\frac{\mu}{2}-m\right) U+(g-f) A U T-\frac{r+\mu}{2} A U+f U T+\frac{r}{2 K} A U T^{2},
\end{aligned}
$$

in terms of the entirely new variables $A=V T^{-1}=S T^{-2}=S(S+I)^{-1} \leq 1, T=\sqrt{S+I}, U=P T^{-1}=$ $P \sqrt{S+I}$, whose respective meanings are the fraction of healthy prey with respect to the total amount of prey, the total prey population on the edge of the herd and the ratio of predators over the total prey population occupying the edge of the area. Extensions are also considered, combining the above with preference for healthy prey consumption and poisonous prey, in which case the last term in the last equation of (8.3) changes sign. As the prey carrying capacity increases, a series of transcritical bifurcations among the various equilibria is discovered, leading ultimately to a supercritical Hopf bifurcation. However note that predators cannot thrive just with infected prey, because the latter are assumed to be too weak to sustain themselves.

The disease could also affect the predators, [18]. After singularity elimination and rescaling, the nondimensional model is:

$$
N^{\prime}=\left(1-N^{2}\right) N-a P, \quad P^{\prime}=P(e a N-m-\lambda Y), \quad Y^{\prime}=Y(\lambda P-n) .
$$

Introducing the new parameter $h=a n \lambda^{-1}$, the predator-free equilibrium is stable for $\rho>1$, the diseasefree one is feasible for $\rho<1$, indicating a transcritical bifurcation, and stable for $h>\rho\left(1-\rho^{2}\right)$ and $\rho>3^{-1 / 2}$. The coexistence equilibrium is feasible for $h \leq 2 \sqrt{3} / 9$, and if the prey population $N_{*}$, solving the quadratic $N^{3}-N+h=0$, is $1>N_{*}>\rho$ and stable for $N_{*}>3^{-1 / 2}$. For $\rho=3^{-1 / 2}$ there is a supercritical Hopf bifurcation at the disease-free equilibrium, i.e. the same that occurs in the purely demographic model.

The purely demographic predator-prey model in [23] presents square root response functions with a Holling type II response; after rescaling it is:

$$
N^{\prime}=N(1-N)-\frac{\sqrt{N} y}{1+a \sqrt{N}}, \quad y^{\prime}=c \frac{\sqrt{N} y}{1+a \sqrt{N}}-s y
$$

The analysis is carried out on the simplified HTI model with $a=0$, with no transformation for removing the singularity at $N=0$, exhibiting bifurcation plots as function of the bifurcation parameter $s$, the predators' mortality, showing that limit cycles are obtained when again the threshold value $3^{-\frac{1}{2}}$ is crossed. Furthermore, the system behavior near the origin is closely analyzed. Using a local nonlinear analysis, the system for $N, y<<1$ becomes:

$$
N^{\prime}=N-\sqrt{N} Z, \quad Z^{\prime}=-s Z .
$$

From the second equation $Z=Z_{0} \exp (-s t)$, with $Z_{0}<<1$. Assuming $N=O\left(Z_{0}^{\alpha}\right)$ there are three possible cases: $\alpha=1$ giving a saddle, $\alpha=2$ and $\alpha>2$. The latter leads to the simplification in the first equation (8.7) which becomes $N^{\prime}=-\sqrt{N} Z$, because $N<<\sqrt{N} Z$. Solving the differential equation, we obtain the parabola $Z=Z_{0}+2 s\left(\sqrt{N}-\sqrt{N_{0} Z_{0}^{\alpha}}\right)$ joining the initial condition $\left(N_{0}, Z_{0}^{\alpha}, Z_{0}\right)$ with the point $\left(0, Z_{0}-2 s \sqrt{N_{0} Z_{0}^{\alpha}}, 0\right)$ on the positive part of the $Z$-axis. Thus the prey is wiped out and as a consequence also the predators follow suit. This is ecologically more realistic than other models that have a saddle in the origin, for which even if the prey are small, they can recover. The intermediate case $\alpha=2$ acts as a threshold, the trajectory goes to the origin. For initial conditions below this "threshold function", the origin acts as a saddle. This fact is also reported in [140]. Simulations reveal that the trajectories 
nevertheless will drift away from the origin, rise up in the phase plane, but ultimately terminate on the $Z$ axis and then, in the absence of prey, also the predators will become extinct.

A related concept is found in [53], where, starting from "first principles", for this see also [111], the predator functional response is derived. Specifically, if $n_{k}$ denotes the density of the group of size $k$ of the prey, the Becker and Döring equations describe the group size dynamics, where $a_{k} n_{1}$ and $b_{k}$ are the rates at which single prey join and leave the group and $K_{1}=K-1$ :

$$
n_{1}^{\prime}=-J_{1}-\sum_{k=1}^{K} J_{k}, \quad n_{k}^{\prime}=J_{k-1}-J_{k}, \quad k=2, \ldots, K_{1}, \quad n_{K}^{\prime}=J_{K_{1}}, \quad J_{k}=a_{k} n_{1} n_{k}-b_{k+1} n_{k+1} .
$$

The total population size is $N=\sum_{k=1}^{K} k n_{k}$. At equilibrium:

$$
\bar{n}_{k}=Q_{k} \bar{n}_{1}^{k}, \quad Q_{k}=\Pi_{i=1}^{k-1} a_{i} b_{i+1}^{-1}, \quad Q_{1}=1, \quad N=\sum_{k=1}^{K} k Q_{k} \bar{n}_{1}^{k} .
$$

Assuming for instance $a_{k}=a \sqrt{k}, b_{k}=b \sqrt{k}$, it follows:

$$
\bar{n}_{k}=\bar{n}_{1}^{k} k^{-\frac{1}{2}} a^{k-1} b^{1-k}, \quad N=\sum_{k=1}^{K} \sqrt{k} a^{k-1} b^{1-k} \bar{n}_{1}^{k} .
$$

Letting $\varphi$ be the predators' functional response with respect to the density of the single prey, the functional response in terms of the total prey $N$ becomes $f(N)=\phi\left(\bar{n}_{1}(N)\right)$. Assuming HTI behavior for the predators, $f(N)=\beta \bar{n}_{1}(N)$, it follows that $f(N) \approx N^{1 / K}$, thus showing a functional response similar to the HTII, even though the individual predator has a HTI response. Deceleration of predation at high prey densities thus depends on the prey rather than on the predator behavior.

In [14] the main finding is that disease weakens group defense and therefore predators thrive. Group defense is here modeled via the type IV functional response $a N\left[a+b N+c N^{2}\right]^{-1}$. This form is derived from the HTII form $a N[1+a h N]^{-1}$ assuming $a \equiv a(N)=a_{0}\left[1+b N^{2}\right]^{-1}$ or $h(N)=h_{0}+h_{N} N$ to take into account increased handling time due to prey population size-dependent group defense. Incorporating different handling times for the susceptible and infected prey, the following two HTIV response functions for the susceptible and infected prey are respectively derived:

$$
\begin{aligned}
& f_{S}=\frac{a_{S} S}{1+a_{S} h_{S 0} S+a_{I} h_{I 0} I+a_{S} h_{S S}^{2} S+\left(a_{S} h_{S I}+a_{I} h_{I S}\right) S I+a_{I} h_{I I} I^{2}}, \\
& f_{I}=\frac{a_{I} I}{1+a_{S} h_{S 0} S+a_{I} h_{I 0} I+a_{S} h_{S S}^{2} S+\left(a_{S} h_{S I}+a_{I} h_{I S}\right) S I+a_{I} h_{I I} I^{2}} .
\end{aligned}
$$

These response functions are simplified by making some assumptions, and then two different disease transmission terms are considered. First, a frequency-dependent tranmission is examined, which yields a separate prevalence equations from the rest of the system, namely $i^{\prime}=i[(\beta-\mu)(1-i)-b]$, and

$$
S^{\prime}=S\left[(b-m)-\mu i-c S-\frac{Z}{1+H_{0} S+H_{S} S^{2}}\right], \quad Z^{\prime}=\frac{S Z}{1+H_{0} S+H_{S} S^{2}}-Z .
$$

Second, the prevalence equation becomes instead $i^{\prime}=i[(\beta S-\mu)(1-i)-b]$ for the density-dependent model. Particular cases lead to the models studied in [13] and in [119]. There are four possible scenarios for the disease-free model, namely: a stable prey-only equilibrium with no coexistence equilibrium; one stable coexistence equilibrium or one unstable coexistence equilibrium with a stable limit cycle surrounding it; bistability of a prey-only and a coexistence equilibrium or bistability between a prey-only and a limit cycle around the unstable coexistence equilibrium; a stable prey-only equilibrium with two unstable coexistence equilibria. 
When the standard incidence ecoepidemic model is considered, predators density at the coexistence equilibrium is reduced when prevalence increases. There is a high risk of extinction for large prey populations, limit cycles are reduced or disappear, replaced by a steady state. Then only the cycles remain, and for even higher values of the prevalence, only the coexistence equilibrium is found. Finally for the largest values of the disease prevalence, the prey-only point is found, followed by disease-induced host extinction, which entails also predators disappearance. Note that host extinction occurs in frequencydependent models, but cannot happen in density-dependent ones.

For the mass action incidence model oscillations and coexistence also occur, but more complex dynamics arise as well. In the density-dependent model indeed coexistence is more complex and contradicts the competitive exclusion principle. Then the same transitions as for the standard incidence model occur, but prevalence may at times decrease when disease transmissibility increases. Further, a cascade of period-doubling bifurcations is discovered leading to chaos and catastrophic behavior of the system.

Finally, incorporation of a saturation effect via a HTII response function within a herd behavior ecoepidemic system with infected predators, [54], gives the model:

$$
\widehat{N}^{\prime}=\widehat{r} \widehat{N}\left(1-\frac{\widehat{N}}{\widehat{K}}\right)-\frac{\widehat{a} \sqrt{\widehat{N}} \widehat{P}}{1+\widehat{T} \widehat{a} \sqrt{\widehat{N}}}, \quad \widehat{P}^{\prime}=\widehat{P}\left[-\widehat{m}+\frac{\widehat{e} \widehat{a} \sqrt{\widehat{N}}}{1+\widehat{T} \widehat{a} \sqrt{\widehat{N}}}-\widehat{\lambda} \widehat{Y}\right], \quad \widehat{Y}^{\prime}=\widehat{Y}[-\widehat{n}+\widehat{\lambda} \widehat{P}] .
$$

The substitution $\widehat{Q}=\sqrt{\widehat{N}}$ and nondimensionalization leads to the following singularity-free model with $Q>0$ :

$$
Q^{\prime}=Q\left(1-\frac{Q^{2}}{a^{2} K T^{2}}\right)-\frac{1}{\lambda} \frac{a^{2} T}{1+Q} P, \quad P^{\prime}=\frac{P}{r}\left[\frac{1}{T} \frac{e}{1+Q} Q-m-Y\right], \quad Y^{\prime}=2 Y[P-n] .
$$

Through a sophisticated bifurcation analysis it is possible to show a wealth of rich behaviors, including catastrophic phenomena and a global bifurcation for which the system collapses completely. Another interesting fact observed in this model is the finite time extinction of the prey, which entails an exponential disapperance of the predators. This new behavior is to be ascribed to the presence of the square root terms in the HTII functional response of (8.11).

\subsection{The Allee effect}

The Allee effect, first introduced in the ecological setting in [3], has been reconsidered in more recent years, [49], also in the context of ecological invasions, [59,92]. It replaces the logistic equation (2.2) by a right hand side with a cubic polynomial with an extra positive root, smaller than the carrying capacity:

$$
N^{\prime}=a N\left(N-a_{0}\right)\left(1-N K^{-1}\right), \quad 0<a_{0}<K .
$$

This entails that for low population values the growth rate becomes negative, so that whenever falling in this situation the population would ultimately be driven to extinction. This phenomenon, due for instance to exogenous activities such as overfishing, is also known in fisheries as critical depensation, [41].

In a purely epidemiological model it has been investigated in [77], but see also [76]. A mass action incidence model with density-dependent incidence is presented, with population-dependent birth and mortality rates $b(N)$ and $m(N)$. With $S$ being the healthy individuals and $\widehat{I}$ the infected, the model is:

$$
S^{\prime}=b(N) N-\beta S \widehat{I}-m(N) S, \quad \widehat{I}^{\prime}=\beta S \widehat{I}-m(N) \widehat{I}-\mu \widehat{I},
$$

where the Allee effect is modeled by $g(N)=b(N)-m(N)=a\left(K_{+}-N\right)\left(N-K_{-}\right), m(N)=a(e N+$ $\left.K_{+} K_{-}+c\right)$ and $b(N)=a\left[-N^{2}+\left(K_{+} K_{-}+e\right) N+c\right]$, in which the quadratic birth rate comes from encounters leading to mating. A suitable reformulation, with $N=S K_{+}^{-1}, I=\widehat{I} K_{+}^{-1}$, leads to:

$$
N^{\prime}=r(1-N)(N-u) N-a I, \quad I^{\prime}=[-\alpha-d-r u+(\sigma-1) N-\sigma I] I, \quad 0<u<\frac{1}{2} .
$$


The following thresholds for persistence and extinction are derived: $R=\sigma N(\alpha+d+r u+N)^{-1}, P_{T}=$ $(\alpha+d+r u)(\sigma-1)^{-1}, R_{0}=\sigma(\alpha+d+r u+1)^{-1}, T_{u}=(u+1)^{3}\left[9\left(u^{2}-u+1\right)\right]^{-1}$, characterizing the number, up to 3 , and the nature of the equilibria. In particular $(1,0)$ is stable for $P_{T}>1$. Bifurcations and limit cycles arise through Hopf bifurcations, that are also studied in the $\alpha-\sigma$ parameter plane, showing cusp catastrophe and transcritical bifurcations, contrary to logistic models, that do not exhibit the same features. It is remarked that small parameter changes may induce possible extinction. Thus density-dependent transmission combined with the Allee effect can lead to host extinction.

In [116] instead the following specific instance of a more general ecoepidemic model is analyzed:

$$
S^{\prime}=S[(1-S-I)-\beta I-a Z], \quad I^{\prime}=I[\beta S-a Z-\mu], \quad Z^{\prime}=Z\left[(b S+\alpha I) \frac{Z}{\theta+Z}-d\right],
$$

where the fraction in the last equation models the weak Allee effect. Technically speaking, the latter means that in the functional response (8.13) we now take the root of the cubic to be negative, $a_{0}<0$. Thus, the population is not bound to become extinguished when its value drops to low values. In (8.16) the behavior of the response function for low population values is similar to the cubic with the above characteristics. The presence of the weak Allee term induces a more complicated disease-free dynamics, with limit cycles and heteroclinic orbits. Further a large value of $\theta$ enhances the predators extinction, but the influence on the disease is negligible, as the model without the Allee effect has the same behavior, coexistence is not possible in both models only if $b<\alpha$, while in the opposite case there are other parameter restrictions for it to occur.

In the very recent paper [86] the following model with strong Allee effect is studied in rescaled form:

$$
S^{\prime}=S[(S-\theta)(1-S-I)-\beta I-a Z], \quad I^{\prime}=I[\beta S-a Z-\mu], \quad Z^{\prime}=Z[a(c S+\gamma I)-d] .
$$

Among the main features, we underline that the model (8.17) exhibits either one, two or three equilibria, corresponding respectively to extinction of all species, competition exclusion or bi-stability and finally tri-stability. Coexistence of the three populations never occurs. If $S(0)<\theta$ the ecosystem is doomed and in presence of bi- or tri-stability the system's outcome depends on the initial conditions. Because of the presence of the Allee effect in the prey, the origin is always an attractor and, under suitable parameter conditions depending on relevant thresholds, it is even a global attactor. In the absence of the coexistence equilibrium, bi-stability occurs either if only susceptible prey is able to survive, when both the disease and predators' reproduction numbers are small, or in case of competitive exclusion, leading to the origin and to another equilibrium, either one of the disease-free or the predator-free points, or the unique stable limit cycle around each one of these points when they are unstable. The unique coexistence equilibrium of system (8.17) is always unstable, when feasible, but then there are three attractors, the origin, and the two disease-free or predator-free points, or alternatively the limit cycles surrounding them. This holds also for the same model without Allee effect, but note that the impossibility of coexistence in such circumstance follows from the assumption that predators cannot distinguish infected and healthy prey, but they are harmed or receive less benefit by consuming the latter. Note also that the parameter $a$ does not appear to influence the feasibility and stability of the above nontrivial equilibria. Finally, competitive exclusion occurs if the thresholds satisfy suitable conditions. Thus the Allee effect renders the system extinction more likely, and entails a more complex dynamics, with limit cycles and heteroclinic orbits. The disease in the Allee model may save the predation-driven extinction, preserving the prey with endemic disease. Instead, predation cannot preserve the disease-driven extinction.

\subsection{Emerging carrying capacity}

In [121] the ecoepidemiological model with disease in the prey is written avoiding explicitly the carrying capacity. In its place it is rather used the concept of "emerging carrying capacity", compare also (8.9). In a certain sense this corresponds to a reuse of the demographic terms as they were originally written 
in the model [130]. We have

$$
\begin{aligned}
& S^{\prime}=S\left[r_{S}-\left(c_{S} S+c_{S I} I\right)-\lambda \frac{I}{S+I}-a \frac{Z}{h+S+I}\right], \\
& I^{\prime}=I\left[r_{I}-\left(c_{I} I+c_{I S} S\right)+\lambda \frac{S}{S+I}-a \frac{Z}{h+S+I}-\mu\right], \quad Z^{\prime}=Z\left(\varepsilon a \frac{S+I}{h+S+I}-m\right) .
\end{aligned}
$$

Working again with the total prey population $N=S+I$ and the disease prevalence $i=I N^{-1}$, the model becomes:

$$
\begin{aligned}
& N^{\prime}=N\left[G(N, i)-a \frac{Z}{h+N}\right], \\
& i^{\prime}=i(1-i)\left[r_{i}-\left(c_{I}-c_{S I}\right) i N-\left(c_{I S}-c_{S}\right)(1-i) N\right], \quad Z^{\prime}=Z\left(\varepsilon a \frac{N}{h+N}-m\right), \\
& G(N, i)=r_{S}(1-i)+\left(r_{I}-\mu\right) i-\left[c_{S}(1-i)^{2}+c_{I} i^{2}+\left(c_{S I}+c_{I S}\right) i(1-i)\right] N,
\end{aligned}
$$

$G$ being the per-capita growth rate, $r_{i}=\lambda-\mu+r_{I}-r_{S}$ the net intrinsic spread rate of the disease prevalence. This disease spread is independent of the predator, while the dynamics of the predator does not depend on the prevalence. A similar model with explicit carrying capacity is indeed (7.9). The equilibria and possible bistability scenarios are analysed as intersections of the nullsurfaces, in the three cases of equal inter- and intra-class competition, $c_{I S}+c_{S I}=c_{I}+c_{S}$, strong inter-class competition, $c_{I S}+c_{S I}>c_{I}+c_{S}$ and weak inter-class competition, $c_{I S}+c_{S I}<c_{I}+c_{S}$. In the latter case, when the system settles at coexistence, increased predator mortality leads to a simultaneous decrease in the infected prey and an increase in predator density, the so-called hydra effect, [120]. It is pointed out that control measures reasonably aimed at reducing the prey to contain the infection could instead lead to ecosystem extinction, both for strong as well as weak inter-class competition. In this framework a richer dynamics appears thus to be possible than using the explicit carrying capacity.

\subsection{Ecogenetic models}

A somewhat related concept to ecoepidemiology is provided by mathematical ecogenetics. In this brief Section we provide a basic introduction. Technically speaking, there is no explicit disease here, but we touch upon this topic because the motivation comes from epidemiology. In fact, there are several situations for which diseases have a genetic origin, or appear only or mainly in the part of a population that harbors a recessive gene. In humans a common such example is the sickle cell anemia.

Models for the mutual effects of a population $Z$ on another one which is genetically distinguished are proposed in $[138,141]$. The main point of these models is that a genetic strain $x$ can give rise to offsprings that are of the other genotype $y$. We illustrate the simplest system below. Here $R$ is the reproduction rate and $p$ and $q=1-p$ are the newborns fractions of type $x$ and $y$ respectively, while $a$ and $b$ represent the intraspecific population pressures exerted on each genetic subpopulation. This is apparent from the first two equations for the prey in the following system:

$$
x^{\prime}=[R p-a x](x+y)-h x Z, \quad y^{\prime}=[R q-b y](x+y)-g y Z, \quad Z^{\prime}=Z[e(h x+g y)-m] .
$$

Evidently, in the above model, the disease transmission by contact is absent, but it is replaced by the reproduction mechanism that produces e.g. new $y$ individuals from the $x$ subpopulation. As a consequence, even if one strain may vanish at some instant, it can be regenerated from the other one. Hence, there is no possibility of finding an equilibrium at which just one of the genetic strains is present. The system exhibits the coexistence and the predator-free equilibria. The latter has population values that are the same found in the predator-free model where, this equilibrium is globally asymptotically stable. The predators can establish themselves permanently in the system, if $R_{Z}=v\left(c+w s^{-1}\right)>1$. They can also destabilize the coexistence equilibrium giving rise to limit cycles, when the reproduction rate $r$ falls below a certain threshold $r^{+}$. 
An almost complete classification of the Routh-Hurwitz stability conditions for a variation of (8.20), including a HTII response function, is further contained in [141], proving to be a guideline for the simulations showing limit cycles. In the very recent paper [50], a further whole continuum of traits is considered, with evolutionary transformations assumed to occur on the same time scale as of ecological processes. The paper shows analytically that in this situation it is possible to stabilize a globally unstable predatorprey system. Also, another important outcome of the investigation is the analytical determination of the Hopf bifurcation curve, rather than exhibiting it as a mere result of numerical simulations.

\subsection{Some other issues}

Other topics that can be considered are here briefly summarized. Models with diverse response functions are presented and their behavior is compared in e.g. [8] and [65]. In [29] migrations of susceptible individuals in ecoepidemic situations are explicitly accounted for. External infection sources or environmental disturbances are explicitly built into the model respectively in [43] and [44]. Symbiotic ecoepidemic models can also be considered, [72]. An ecoepidemic model formulated by means of cellular automata has been introduced in [51].

The following model for the ecology of the Salton Sea, [33], has been proposed. It is a prototype of several other subsequent investigations, e.g. [64]. It accounts for the Tilapia fish, Oreochromis mossambicus, which may be infected by bacteria, causing Avian botulism leading ultimately to the death of their predators, the pelican birds:

$$
S^{\prime}=r S\left[1-\frac{S+I}{K}-\lambda I\right], \quad I^{\prime}=I\left[\lambda S-\frac{m Z}{a+I}-\mu\right], \quad Z^{\prime}=Z\left[\frac{\theta I}{a+I}-d\right] .
$$

Global asymptotic stability of the coexistence state is shown with a suitable Lyapunov function whenever this equilibrium is feasible, provided the renewal rate is bounded from above. Numerical experiments indicate also the existence of sustained population oscillations. For global stability results on a similar model with a highly nonlinear incidence function, of the form $\lambda I^{p} S^{q}$, see also [145].

In [13] the models, similar to (4.2) but for the presence of the mass action incidence used here, are considered:

$$
\begin{aligned}
& N^{\prime}=N\left[r(1-N)-\frac{Z}{h+N}\right], \quad Z^{\prime}=Z\left[\frac{N}{h+N}-m-\mu i_{Z}\right], \\
& i_{Z}^{\prime}=i_{Z}\left[(\beta Z-\mu)\left(1-i_{Z}\right)-\frac{N}{h+N}\right],
\end{aligned}
$$

for the disease in the predators and

$$
N^{\prime}=N\left[r(1-N)-\frac{Z}{h+N}-\mu N i_{N}\right], \quad i_{N}^{\prime}=i_{N}\left[(\beta N-\mu)\left(1-i_{N}\right)-r\right], \quad Z^{\prime}=Z\left[\frac{N}{h+N}-m\right]
$$

for the epidemics in the prey. Denoting by a bar the time average of the populations over a period $T$ of the limit cycle, say $\bar{Z}=T^{-1} \int_{0}^{T} Z d t$, a basic reproduction number based on the time average of oscillations is introduced $\bar{R}_{0}=\beta \bar{Z}(\mu+m)^{-1}$ for (8.22) and $\bar{R}_{0}=\beta \bar{N}(\mu+r)^{-1}$ for (8.23) to explain the endemicity of diseases in case of oscillatory dynamics. This differs from the corresponding (unstable) equilibriumbased basic reproductive number $R_{0}^{*}$. This concept explains the remark that endemic thresholds differ for equilibria and oscillatory behaviors, [91].

The findings indicate that for (8.22), the system remains disease-free for a larger parameter range due to the persistent oscillations. For (8.23) instead, even though $R_{0}^{*}<1$, the disease remains endemic. This shows that it becomes endemic for values of the contact rate smaller than can be expected from the standard value of $R_{0}^{*}$. These results entail relevant consequences for the strategies of disease eradication and must therefore be seriously taken into account. For instance, when the disease affects the predators, their indiscriminate culling will increase the time average of predator density, the hydra effect, [120], 
and therefore produce opposite results of those expected. Instead, causing endogenous oscillations in the system, e.g. by increasing prey abundance through a larger carrying capacity, may lead to $\bar{R}_{0}<1$, ensuring disease eradication. For a disease affecting the prey, oscillations instead render the disease more difficult to fight, and policies aiming at stabilizing the system should be favored, such as reducing the prey carrying capacity.

Finally, both this model (8.22) as well as (4.2) are further investigated in [12], to show rich dynamics including turning points, saddle-node and subcritical Hopf bifurcations, branching of periodic solutions, period doubling route to chaos, regions of bi- and tristability involving an endemic limit cycle, an endemic equilibrium and a disease-free limit cycle.

\section{Concluding remarks}

Most likely every living organism is either affected by some kind of malaise, if not a real disease, or infected by some kind of parasite; we can just think of our intestinal flora, for instance. This consideration alone should suggest that in ecology the proper view of population interactions cannot be adequate if these conditions, that at first sight might be overlooked or considered marginal, are not seriously taken into account and formally imbedded in the ecosystem descriptions. The very first example (3.1) shows us the wrong conclusions one would infer, if the disease is not suitably modeled: recall the analysis of condition (3.2) compared to the corresponding one (2.3) of the purely demographic model (2.1).

Ecoepidemiology is a fast growing, interesting and fascinating field. In this paper we have tried to elucidate some of the relevant research directions, outlining some of the many contributions that have appeared in the past twenty-five years in this field. The contributions of several researchers around the world have helped in its building and shaping along the years, stimulating the debate with deeper ideas and more sophisticated models than the first ones that were formulated. In our opinion it is a living example of how, starting from a simple idea, an avalanche of new concepts can be developed when scientists from possibly different backgrounds share ideas, thereby mutually fertilizing their investigations.

This review cannot be comprehensive: we apologize for the works that have been omitted, this in part also being due to lack of space. From the references provided however, further papers can be retrieved. In particular the deliberate choice has been made of not discussing the space-related models, see [97] for some aspects of them. These ecosystems formulated in space lead to pattern formation, invasions and a wealth of other interesting phenomena, for which another suitably focused review would be necessary. We hope nevertheless to have transmitted to the reader the flavor of, and some of our feelings for, this research field, thereby arousing her/his curiosity and perhaps stimulating her/him to contribute to these topics in continuous development.

Acknowledgments: The author expresses his sincerest warm thanks to A. Morozov and V. Volpert for their invitation to write this paper, their patience during its preparation and for their valuable comments on its careful rereading, which allowed a much improved presentation. The author also warmly thanks F. Hilker for his constructive suggestions in critically reading the final version of the manuscript.

\section{References}

[1] V. Ajraldi, M. Pittavino, E. Venturino. Modelling herd behavior in population systems. Nonlinear Analysis Real World Applications, 12 (2011), 2319-2338.

[2] H. R., Akcakaya. Population cycles of mammals: evidence for ratio-dependent predator-prey hypothesis. Ecol. Monogr., 62 (1992) 119-142.

[3] W. C. Allee. The Social Life of Animals. New York: Norton and Co. (1938).

[4] R. M. Anderson, R. M. May. The invasion, persistence and spread of infectious diseases within animal and plant communities. Philos. Trans. R. Soc. London B, 314 (1986), 533-570.

[5] O. Arino, M. Delgado, M. Molina-Becerra. Asymptotic behaviour of disease-free equilibriums of an age-structured predator-prey model with disease in the prey. Discrete and Continuous Dynamical Systems Series B, 4 (2004), 501-515.

[6] R. A. Armstrong, R. McGehee. Competitive exclusion. The American Naturalist, 115 (1980), 151-170. 
[7] P. Auger, R. Mchich, T. Chowdhury, G. Sallet, M. Tchuente, J. Chattopadhyay. Effects of a disease affecting a predator on the dynamics of a predator-prey system. Journal of Theoretical Biology, 258 (2009), 344-351.

[8] N. Bairagi, P.K. Roy, J. Chattopadhyay. Role of infection on the stability of a predator-prey system with several response functions-A comparative study. Journal of Theoretical Biology, 248 (2007), 10-25.

[9] N. Bairagi, S. Chaudhuri, J. Chattopadhyay. Harvesting as a disease control measure in an eco-epidemiological system - A theoretical study. Mathematical Biosciences, 217 (2009), 134-144.

[10] N. Bairagi, R.R. Sarkar, J. Chattopadhyay. Impacts of incubation delay on the dynamics of an eco-epidemiological system-A theoretical study. Bulletin of Mathematical Biology, 70 (2008), 2017-2038. DOI: 10.1007/s11538-008-9337-y

[11] M. Banerjee, E. Venturino. A phytoplankton-toxic phytoplankton-zooplantkon model. Ecological Complexity, 8 (2011), 239-248.

[12] A. M. Bate, F. M. Hilker. Complex dynamics in an eco-epidemiological model. Bull. Math. Biol., 75 (2013), $2059-2078$. DOI: $10.1007 / \mathrm{s} 11538-013-9880-\mathrm{z}$

[13] A. M. Bate, F. M. Hilker. Predator-prey oscillations can shift when diseases become endemic. Journal of Theoretical Biology, 316 (2013), 1-8.

[14] A. M. Bate, F. M. Hilker. Disease in group-defending prey can benefit predators. Theor. Ecol., 7 (2014), 87-100. DOI: $10.1007 / \mathrm{s} 12080-013-0200-\mathrm{x}$

[15] S. Bhattacharyya, D. K. Bhattacharya. Pest control through viral disease: mathematical modeling and analysis. J. Theor. Biol., 238 (2006), 177-196.

[16] J. Beddington. Mutual interference between parasites or predators and its effect on searching efficiency. J.Anim. Ecol., $51(1975), 331-340$.

[17] E. Beltrami, T.O. Carroll. Modelling the role of viral disease in recurrent phytoplankton blooms. J. Math. Biol., 32 (1994), 857-863.

[18] S. Belvisi, E. Venturino. An ecoepidemic model with diseased predators and prey group defense. SIMPAT, 34 (2013), 144-155. DOI: 10.1016/j.simpat.2013.02.004

[19] E. Beretta, Y. Kuang. Modeling and analysis of a marine bacteriophage infection. Math. Biosci., 149 (1998), 57-76.

[20] A. Berruti, V. La Morgia, E. Venturino, S. Zappalà. Competition among invasive and native species: the case of European and mountain hares, CMMSE 14, July 3rd-7th, 2014, Costa Ballena, Rota, Cádiz (Spain), (J. Vigo-Aguiar, I.P. Hamilton, J. Medina, P. Schwertfeger, W. Sproessig, M. Demiralp, E. Venturino, V.V. Kozlov, P. Oliveira Editors) v. I, 170-181.

[21] F. Bianco, E. Cagliero, M. Gastelurrutia, E. Venturino. Metaecoepidemic models: infected and migrating predators. Int. J. Comp. Math., 89(13-14) (2012), 1764-1780.

[22] C. Bosica, A. De Rossi, N. L. Fatibene, M. Sciarra, E. Venturino. Two-strain ecoepidemic systems: the obligated mutualism case. Applied Math. Inf. Sci. 9(4), (2015) 1677-1685.

[23] P. A. Braza. Predator-prey dynamics with square root functional responses. Nonlinear Analysis: Real World Applications, 13 (2012), 1837-1843.

[24] I. M. Bulai, R. Cavoretto, B. Chialva, D. Duma, E. Venturino. Comparing disease control policies for interacting wild populations. Nonlinear Dynamics, 79 (2015), 1881-1900.

[25] S. Busenberg, P. van den Driessche. Analysis of a disease transmission model in a population with varying size. J. of Math. Biology, 28 (1990), 257-270.

[26] E. Cagliero, E. Venturino. Ecoepidemics with infected prey in herd defence: the harmless and toxic cases. International Journal of Computer Mathematics, (2015), to appear. DOI: 10.1080/00207160.2014.988614

[27] R. Cavoretto, S. Collino, B. Giardino, E. Venturino. A two-strain ecoepidemic competition model. Theoretical Ecology, 8(1) (2015), 37-52. DOI: 10.1007/s12080-014-0232-x

[28] R. Cavoretto, A. De Rossi, E. Perracchione, E. Venturino, Reliable approximation of separatrix manifolds in competition models with safety niches, to appear in International Journal of Computer Mathematics.

[29] S. Chatterjee, J. Chattopadhyay. Role of migratory bird population in a simple eco-epidemiological model. Mathematical and Computer Modelling of Dynamical Systems: Methods, Tools and Applications in Engineering and Related Sciences, 13 (2007), 99-114. DOI: 10.1080/13873950500303352

[30] S. Chatterjee, K. Das, J. Chattopadhyay. Time delay factor can be used as a key factor for preventing the outbreak of a disease-Results drawn from a mathematical study of a one season eco-epidemiological model. Nonlinear Analysis: Real World Applications, 8 (2007), 1472-1493.

[31] S. Chatterjee, M. Isaia, E. Venturino. Spiders as biological controllers in the agroecosystem. Journal of Theoretical Biology 258 (2009), 352-362.

[32] J. Chattopadhyay, O. Arino. A predator-prey model with disease in the prey. Nonlinear Analysis, 36 (1999), $747-766$.

[33] J. Chattopadhyay, N. Bairagi. Pelicans at risk in Salton sea - an eco-epidemiological model. Ecological Modelling, 136 (2001), 103-112.

[34] J. Chattopadhyay, S. Chatterjee, E. Venturino. Patchy agglomeration as a transition from monospecies to recurrent plankton blooms. Journal of Theoretical Biology, 253 (2008), 289-295.

[35] J. Chattopadhyay, R.R. Sarkar, G. Ghosal. Removal of infected prey prevent limit cycle oscillations in an infected prey-predator system - a mathematical study. Ecological Modelling, 156 (2002), 113-121.

[36] J. Chattopadhayay, R. R. Sarkar, S. Mandal. Toxin-producing Plankton May Act as a Biological Control for Planktonic Blooms-Field Study and Mathematical Modelling. J. Theor. Biol., 215 (2002), 333-344. doi:10.1006/jtbi.2001.2510

[37] J. Chattopadhyay, R. R. Sarkar, S. Pal. Dynamics of nutrient-phytoplankton interaction in the presence of viral infection. BioSystems, 68 (2003), 5-17. 
[38] S. Chaudhuri, A. Costamagna, E. Venturino. Epidemics spreading in predator-prey systems. Int. J. Comp. Math., 89 (2012), 561-584.

[39] S. Chaudhuri, J. Chattopadhyay, E. Venturino. Toxic phytoplankton-induced spatiotemporal patterns. J. of Biological Physics, 38 (2012), 331-348.

[40] S. Chaudhuri, A. Costamagna, E. Venturino. Ecoepidemics overcoming the species-barrier and being subject to harvesting. Mathematical Medicine and Biology, 30 (2013), 73-93. doi:10.1093/imammb/dqr026

[41] C. Clark. Mathematical bioeconomics: the optimal management of renewable resources. Wiley, New York, (1976).

[42] C. Cosner, D. L. De Angelis. Effects of spatial grouping on the functional response of predators. Theoretical Population Biology, 56 (1999), 65-75.

[43] K. p. Das, S. Roy, J. Chattopadhyay. Effect of disease-selective predation on prey infected by contact and external sources. BioSystems, 95 (2009), 188-199

[44] K. p. Das, J. Chattopadhyay. Role of environmental disturbance in an eco-epidemiological model with disease from external source. Math. Meth. Appl. Sci., 35 (2012), 659-675

[45] K. p. Das, K. Kundu, J. Chattopadhyay. A predator-prey mathematical model with both the populations affected by diseases. Ecological Complexity, 8 (2011), 68-80.

[46] D. De Angelis, R. Goldstein, R. and O'Neill. A model for trophic interaction. Ecology, 56 (1975), 881-892.

[47] A. De Rossi, F. Lisa, L. Rubini, A. Zappavigna, E. Venturino. A food chain ecoepidemic model: infection at the bottom trophic level. Ecological Complexity 21 (2015) 233-245.

[48] M. Delgado, M. Molina-Becerra, A. Suarez. Relating disease and predation: equilibria of an epidemic model. Math. Methods Appl. Sci., 28 (2005), 349-362.

[49] B. Dennis. Allee effects: population growth, critical density, and the chance of extinction. Nat. Res. Model., 3 (1989), $481-538$

[50] J. Z. Farkas, A. Y. Morozov. Modelling Effects of Rapid Evolution on Persistence and Stability in Structured PredatorPrey Systems. Math. Model. Nat. Phenom., 9(3) (2014), 26-46. doi: 10.1051/mmnp/20149303

[51] L. Ferreri, E. Venturino. Cellular automata for contact ecoepidemic processes in predator-prey systems. Ecological Complexity, 13 (2013), 8-20.

[52] Q. L. Gao, H. W., Hethcote. Disease transmission models with density dependent demographics. J. Math. Biol., 30 (1992), 717-731.

[53] S. A. H. Geritz, M. Gyllenberg. Group defence and the predator's functional response. J. Math. Biol., 66 (2013), 705-717. DOI: 10.1007/s00285-012-0617-7

[54] G. Gimmelli, B. W. Kooi, E. Venturino. Ecoepidemic models with prey group defense and feeding saturation. Ecological Complexity, 22 (2015), 50-58.

[55] E. González-Olivares, R. Ramos-Jiliberto. Dynamic consequences of prey refuges in a simple model system: more prey, fewer predators and enhanced stability. Ecological Modelling, 166 (2003), 135-146.

[56] E. González-Olivares, R. Ramos-Jiliberto. Comments to the effect of prey refuge in a simple predator-prey model. Ecological Modelling, 232 (2012), 158-160.

[57] D. Greenhalgh, M. Haque. A predator-prey model with disease in the prey species only. Math. Meth. Appl. Science, 30 (2007), 911-929.

[58] M. E. Gurtin, R. C. McCamy. Nonlinearly age-dependent population dynamics. Archs. Ration. Mech. Analysis, 54 (1974), 281-300.

[59] M. Gyllenberg, J., Hemminki, T. Tammaru. Allee effects can both conserve and create spatial heterogeneity in population densities. Theor. Pop. Biol., 56 (1999), 231-242.

[60] K.P. Hadeler, H.I.Freedman. Predator-prey population with parasitic infection. J. Math. Biol., 27 (1989), 609-631.

[61] L. Han, Z. Ma, H.W. Hethcote. Four predator prey models with infectious diseases. Math. Comp. Modelling, 30 (2001), 849-858.

[62] I. Hanski, M. Gilpin (Editors) Metapopulation biology: ecology, genetics and evolution, Academic Press, London (1997).

[63] I. Hanski, A. Moilanen, T. Pakkala, M. Kuussaari. Metapopulation persistence of an endangered butterfly: a test of the quantitative incidence function model. Conservation Biology, 10 (1996), 578-590.

[64] M. Haque, J. Chattopadhyay. Influences of non-linear incidence rate in an eco-epidemiological model of the Salton Sea. Nonlinear Studies, 10 (2003), 373-388.

[65] M. Haque, S. Rahman, E. Venturino. Comparing functional responses in predator-infected eco-epidemics models. BioSystems, 114 (2013), 98-117.

[66] M. Haque, S. Sarwardi, S. Preston, E. Venturino. Effect of delay in a Lotka-Volterra type predator-prey model with a transmissible disease in the predator species. Mathematical Biosciences, 234 (2011), 47-57.

[67] M. Haque, J. Zhen, E. Venturino. An epidemiological predator-prey model with standard disease incidence. Mathematical Methods in the Applied Sciences, 32 (2009), 875-898.

[68] M. Haque, E. Venturino. The role of transmissible diseases in the Holling-Tanner predator-prey model. Theoretical Population Biology, 70 (2006), 273-288.

[69] M. Haque, E. Venturino. Increase of the prey may decrease the healthy predator population in presence of a disease in the predator. HERMIS, 7 (2006), 39-60.

[70] M. Haque, E. Venturino. An ecoepidemiological model with disease in the predator: the ratio-dependent case. Math. Meth. Appl. Sci., 30 (2007), 1791-1809.

[71] M. Haque, E. Venturino. Effect of parasitic infection in the Leslie-Gower predator-prey model. Journal of Biological Systems, 16 (2008), 445-461. 
[72] M. Haque, E. Venturino. Mathematical models of diseases spreading in symbiotic communities. in J.D. Harris, P.L. Brown (Editors), Wildlife: Destruction, Conservation and Biodiversity, NOVA Science Publishers, New York, (2009) $135-179$.

[73] H. W. Hethcote. The mathematics of infectious diseases. SIAM Review, 42 (2000), 599-653.

[74] H. W. Hethcote, H. W. Stech, and P. van den Driessche. Periodicity and stability in epidemic models: A survey. In Differential Equations and Applications in Ecology, Epidemics and Population Problems, S. N. Busenberg and K. L. Cooke, eds., Academic Press, New York (1981), 65-82.

[75] H.W. Hethcote, W. Wang, L. Han, Z. Ma. A predator prey model with infected prey. Theoretical Population Biology, 66 (2004), 259-268.

[76] F. M. Hilker. Population collapse to extinction: the catastrophic combination of parasitism and Allee effect. Journal of Biological Dynamics, 4 (2010), 86-101.

[77] F. M. Hilker, M. Langlais, H. Malchow. The Allee Effect and Infectious Diseases: Extinction, Multistability, and the (Dis-)Appearance of Oscillations. The American Naturalist, 173 (2009), 72-88.

[78] F. M. Hilker, H. Malchow. Strange Periodic Attractors in a Prey-Predator System with Infected Prey. Mathematical Population Studies, 13 (2006), 119-134. DOI: 10.1080/08898480600788568

[79] F. M. Hilker, H. Malchow, M. Langlais, S. V. Petrovskii. Oscillations and waves in a virally infected plankton system: Part II: Transition from lysogeny to lysis. Ecological Complexity, 3 (2006), 200-208.

[80] F. Hilker, K. Schmitz. Disease-induced stabilization of predator-prey cycles. Journal of Theoretical Biology, 255 (2008), 299-306.

[81] I. S. Hotopp, H. Malchow, E. Venturino. Switching feeding among sound and infected prey in ecoepidemic systems. Journal of Biological Systems, 18 (2010), 727-747. DOI: 10.1142/S0218339010003718.

[82] Y.H. Hsieh, C.K. Hsiao. A predator-prey model with disease infection in both populations. Mathematical Medicine and Biology, 25 (2008), 247-266.

[83] S. Jana, T. K. Kar. Modeling and analysis of a prey-predator system with disease in the prey. Chaos, Solitons \& Fractals, 47 (2013), 42-53.

[84] S. Jana, T.K. Kar. A mathematical study of a prey-predator model in relevance to pest control. Nonlinear Dynamics, 74 (2013), 667-683.

[85] A. Kacha, M. H. Hbid, R. Bravo. Mathematical study of bacteria-fish model with level of infection structure. Nonlinear Analysis: Real World Applications, 10 (2009), 1662-1678.

[86] Y. Kang, S. K. Sasmal, A. M. Bhowmick, J. Chattopadhyay. Dynamics of a predator-prey system with prey subject to Allee effects and disease. Mathematical Biosciences and Engineering, 11 (2014), 877-918.

[87] T. K. Kar, S. Jana. Application of three controls optimally in a vector-borne disease-a mathematical study. Commun. Nonlinear Sci. Numer. Simul. 18 (2013), 2868-2884.

[88] T. K. Kar, S. Jana. A theoretical study on mathematical modelling of an infectious disease with application of optimal control. BioSystems 111 (2013), 37-50.

[89] Q.J.A. Khan, E. Balakrishnan, G.C. Wake. Analysis of a predator-prey system with predator switching. Bull. Math. Biol., 66 (2004), 109-123.

[90] Q.J.A. Khan, B.S. Bhatt, R.P. Jaju. Switching model with two habitats and a predator involving group defence. J. of Nonlinear Mathematical Physics, 5 (1998), 212-223.

[91] B. W., Kooi, G. A. K., van Voorn, K. p. Das. Stabilization and complex dynamics in a predator-prey model with predator suffering from an infectious disease. Ecol. Complexity, 8 (2011), 113-122.

[92] M. A. Lewis, P. Kareiva. Allee dynamics and the spread of invading organisms. Theor. Popul. Biol., 43 (1993), $141-158$.

[93] J. Liu. Stability and Hopf bifurcation in a prey-predator system with disease in the prey and two delays. Abstract and Applied Analysis, (2014), Article ID 624546, 15 pages. DOI: 10.1155/2014/624546

[94] Z. Ma, S. Wang, Z. Li. The effect of prey refuge in a simple predator-prey model. Ecological Modelling, 222 (2011), 3453-3454.

[95] H. Malchow H, F. M. Hilker, S. V. Petrovskii, K. Brauer Oscillations and waves in a virally infected plankton system: Part I: The lysogenic stage. Ecological Complexity, 1 (2004), 211-223.

[96] H. Malchow, F. M. Hilker, R. R. Sarkar, K. Brauer. Spatiotemporal patterns in an excitable plankton system with lysogenic viral infection. Mathematical and Computer Modelling, 42 (2005), 1035-1048.

[97] H. Malchow, S. Petrovskii, E. Venturino. Spatiotemporal patterns in Ecology and Epidemiology. CRC, Boca Raton, (2008).

[98] J. Mena-Lorca, H. W. Hethcote. Dynamic models of infectious diseases as regulator of population sizes. J. Math. Biology, 30 (1992), 693-716.

[99] A. Molter, M. Rafikov. Nonlinear optimal control of population systems: applications in ecosystems. Nonlinear Dynamics, 76 (2014), 1141-1150.

[100] M. Rafikov, J. C. Silveira. On dynamical behavior of the sugarcane borer - Parasitoid agroecosystem, Ecological Complexity, 18 (2014), 67-73.

[101] A. Morozov. Revealing the role of predator-dependent disease transmission in the epidemiology of a wildlife infection: a model study. Theoretical Ecology, 5 (2012), 517-532.

[102] A.Y. Morozov. Emergence of Holling type III zooplankton functional response: Bringing together field evidence and mathematical modelling. Journal of Theoretical Biology, 265 (2010), 45-54.

[103] N. M. Oliveira, F. M. Hilker. Modelling Disease Introduction as Biological Control of Invasive Predators to Preserve Endangered Prey. Bulletin of Mathematical Biology, 72 (2010), 444-468. DOI: 10.1007/s11538-009-9454-2 
[104] S. Palomino Bean, A.C.S. Vilcarromero, J.F.R. Fernandes, O. Bonato. Co-existência de Espécies em Sistemas Presapredador com Switching (Species coexistence in predator-prey systems with switching). TEMA Tend. Mat. Apl. Comput., 7 (2006), 317-326.

[105] E. Renshaw. Modelling biological populations in space and time. Cambridge Univ. Press, Cambridge, UK (1991).

[106] M.G. Roberts, J.A.P. Heesterbeek. Characterizing the next-generation matrix and basic reproduction number in ecological epidemiology. J. Math. Biol., 66 (2013), 1045-1064, DOI: 10.1007/s00285-012-0602-1

[107] T. Romano, M. Banerjee, E. Venturino. A comparison of several plankton models for red tides. in G. Kehayias (Editor) Zooplankton: Species Diversity, Distribution and Seasonal Dynamics, Nova Science Publishers, Hauppauge, NY, 2014, 19-63. ISBN: 978-1-62948-720-5

[108] M.L. Rosenzweig, R.H. MacArthur. Graphical representation and stability conditions of predator-prey interactions. Am. Nat., 97 (1963), 209-223.

[109] S. Roy, S. Alam, J. Chattopadhyay. Competitive effects of toxin-producing phytoplankton on overall plankton populations in the Bay of Bengal. Bull. Math. Biol., 68 (2006), 2303-2320.

[110] S. Roy, J. Chattopadhyay. Disease-selective predation may lead to prey extinction. Math. Meth. Appl. Sci., 28 (2005), 1257-1267.

[111] G.D. Ruxton. Short term refuge use and stability of predator-prey models. Theoretical Population Biology, 47 (1995), $1-17$.

[112] R.A. Saenz, H.W. Hethcote. Competing species models with an infectious disease. Mathematical Biosciences and Engineering, 3 (2006), 219-235.

[113] R.R.Sarkar, S.Pal, J.Chattopadhyay. Role of two toxin-producing plankton and their effect on phytoplanktonzooplankton system, a mathematical study supported by experimental findings. BioSystems, 80 (2005), 11-13.

[114] S. Sarwardi, M. Haque, E. Venturino. A Leslie-Gower Holling-type II ecoepidemic model. J. Applied Mathematics and Computing, 35 (2011), 263-280. DOI: 10.1007/s12190-009-0355-1

[115] S. Sarwardi, M. Haque, E. Venturino. Global stability and persistence in Leslie-Gower Holling type II diseased predator ecosystems. J. Biol. Phys., 37 (2011), 91-106. DOI: 10.1007/s10867-010-9201-9.

[116] S. K. Sasmal, J. Chattopadhyay. An eco-epidemiological system with infected prey and predator subject to the weak Allee effect. Mathematical Biosciences, 246 (2013), 260-271.

[117] M. Semplice, E. Venturino. Travelling waves in plankton dynamics. Math. Model. Nat. Phenom., 8 (2013), No. 6, 64-79. DOI: $10.1051 / \mathrm{mmnp} / 20138605$.

[118] M. Sen, E. Venturino. A model for which toxic and non-toxic phytoplankton are indistinguishable by the zooplantkon. AIP Conf. Proc. 1479, ICNAAM 2012 (2012), T. Simos, G. Psihoylos, Ch. Tsitouras, Z. Anastassi (Editors), $1315-1318$. doi: $10.1063 / 1.4756397$

[119] M. Sieber, F. M. Hilker. Prey, predators, parasites: intraguild predation or simpler community modules in disguise? Journal of Animal Ecology, 80 (2011), 414-421. doi: 10.1111/j.1365-2656.2010.01788.x

[120] M. Sieber, F. M. Hilker. The hydra effect in predator-prey models. J. Math. Biol., 64 (2012), 341-360.

[121] M. Sieber, H. Malchow, F. M. Hilker. Disease-induced modification of prey competition in eco-epidemiological models. Ecological Complexity, 18 (2014), 74-82.

[122] I. Siekmann, H. Malchow, E. Venturino. An extension of the Beretta-Kuang model of viral diseases. Mathematical Biosciences and Engineering, 5 (2008), 549-565.

[123] I. Siekmann, H. Malchow, E. Venturino. On competition of predators and prey infection, Ecological Complexity, 7 (2010), 446-457; doi:10.1016.

[124] B.K. Singh, J. Chattopadhyay, S. Sinha. The role of virus infection in a simple phytoplankton zooplankton system. Journal of Theoretical Biology, 231 (2004), 153-166.

[125] D. Stiefs, E. Venturino, U. Feudel. Evidence of chaos in ecoepidemic models. Mathematical Biosciences and Engineering, 6 (2009), 855-871.

[126] C. Tannoia, E. Torre, E. Venturino. An incubating diseased-predator ecoepidemic model. J. Biol. Phys., 38 (2012), $705-720$.

[127] M. Tansky. Switching effects in prey-predator system. J. Theor. Biol., 70 (1978), 263-271.

[128] R.K. Upadhyay, N. Bairagi, K. Kundu, J. Chattopadhyay. Chaos in eco-epidemiological problem of the Salton Sea and its possible control. Applied Mathematics and Computation, 196 (2008), 392-401.

[129] E. Venturino. The influence of diseases on Lotka-Volterra systems. Rocky Mountain J. of Mathematics, 24 (1994), 381-402.

[130] E. Venturino. Epidemics in predator-prey models: disease in the prey. in Mathematical Population dynamics, Analysis of heterogeneity 1, in O. Arino, D. Axelrod, M. Kimmel, M. Langlais (Editors) (1995) 381-393.

[131] E. Venturino. The effects of diseases on competing species. Math. Biosc., 174 (2001), 111-131.

[132] E. Venturino. Epidemics in predator-prey models: disease in the predators. IMA J. Math. Appl. Med. and Biol., 19 (2002), 185-205.

[133] E. Venturino. A stage-dependent ecoepidemic model. WSEAS Transactions on Biology and Biomedicine, 1 (2004), 449-454.

[134] E. Venturino. How diseases affect symbiotic communities. Math. Biosc., 206 (2007), 11-30.

[135] E. Venturino. Ecoepidemic models with disease incubation and selective hunting. Journal of Computational and Applied Mathematics, 234 (2010), 2883-2901.

[136] E. Venturino. A minimal model for ecoepidemics with group defense. J. of Biological Systems, 19 (2011), $763-785$. 
[137] E. Venturino. Simple metaecoepidemic models. Bulletin of Mathematical Biology, 73 (2011), 917-950.

[138] E. Venturino. An ecogenetic model. Appl. Math. Letters, 25 (2012), 1230-1233.

[139] E. Venturino, M. Isaia, F. Bona, S. Chatterjee, G. Badino. Biological controls of intensive agroecosystems: wanderer spiders in the Langa Astigiana. Ecological Complexity, 5 (2008), 157-164.

[140] E. Venturino, S. Petrovskii. Spatiotemporal Behavior of a Prey-Predator System with a Group Defense for Prey. Ecological Complexity, 14 (2013), 37-47. doi: 10.1016/j.ecocom.2013.01.004

[141] C. Viberti, E. Venturino. An ecosystem with Holling type II response and predators' genetic variability. Mathematical Modelling and Analysis, 19, (2014) 371-394.

[142] P. Waltman. Competition models in population biology. SIAM, Philadelphia, 1983.

[143] Y. Wang, J. Wang. Influence of prey refuge on predator-prey dynamics. Nonlinear Dynamics, 67 (2012), $191-201$.

[144] J. A. Wiens. Metapopulation dynamics and landscape ecology, in I. A. Hanski, M. E. Gilpin (Ed.s), Metapolulation Biology: Ecology, Genetics and Evolution, San Diego: Academic Press (1997) 43-62.

[145] J. Zhen, M. Haque. Global stability analysis of an eco-epidemiological model of the Salton sea. Journal of Biological Systems, 14 (2006), 373-385. 